\title{
STABILITY OF CLUSTER SOLUTIONS IN A COOPERATIVE CONSUMER CHAIN MODEL
}

\author{
JUNCHENG WEI AND MATTHIAS WINTER
}

\begin{abstract}
We study a cooperative consumer chain model which consists of one producer and two consumers. It is an extension of the Schnakenberg model suggested in $[9,22]$ for which there is only one producer and one consumer. In this consumer chain model there is a middle component which plays a hybrid role: it acts both as consumer and as producer. It is assumed that the producer diffuses much faster than the first consumer and the first consumer much faster than the second consumer. The system also serves as a model for a sequence of irreversible autocatalytic reactions in a container which is in contact with a well-stirred reservoir.

In the small diffusion limit we construct cluster solutions in an interval which have the following properties: The spatial profile of the third component is a spike. The profile for the middle component is that of two partial spikes connected by a thin transition layer. The first component in leading order is given by a Green's function. In this profile multiple scales are involved: The spikes for the middle component are on the small scale, the spike for the third on the very small scale, the width of the transition layer for the middle component is between the small and the very small scale. The first component acts on the large scale. To the best of our knowledge, this type of spiky pattern has never before been studied rigorously. It is shown that, if the feedrates are small enough, there exist two such patterns which differ by their amplitudes.

We also study the stability properties of these cluster solutions. We use a rigorous analysis to investigate the linearized operator around cluster solutions which is based on nonlocal eigenvalue problems and rigorous asymptotic analysis. The following result is established: If the time-relaxation constants are small enough, one cluster solution is stable and and the other one is unstable. The instability arises through large eigenvalues of order $O(1)$. Further, there are small eigenvalues of order $o(1)$ which do not cause any instabilities.
\end{abstract}

Our approach requires some new ideas:

(i) The analysis of the large eigenvalues of order $O(1)$ leads to a novel system of nonlocal eigenvalue problems with inhomogeneous Robin boundary conditions whose stability properties have been investigated rigorously.

(ii) The analysis of the small eigenvalues of order $o(1)$ needs a careful study of the interaction of two small length scales and is based on a suitable inner/outer expansion with rigorous error analysis. It is found that the order of these small eigenvalues is given by the smallest diffusion constant $\epsilon_{2}^{2}$.

Pattern Formation, Reaction-Diffusion System, Consumer Chain Model, Cluster Solutions, Stability.

Department of Mathematics, The Chinese University of Hong Kong, Shatin, Hong Kong (wei@math. cuhk.edu.hk).

Brunel University, Department of Mathematical Sciences, Uxbridge UB8 3PH, United Kingdom (matthias.winter@brunel.ac.uk). 
Primary 35B35, 92C40; Secondary 35B40

\section{Chain models in Biology And other sciences}

Models involving a chain of components play an important role in biology, chemistry, social sciences and many other fields. Well-known examples include food chains, consumer chains, genetic signaling pathways and autocatalytic chemical reactions or nuclear chain reactions. For food chains it is commonly assumed that there is only limited supply of resources which leads to a saturation effect. On the other hand, for autocatalytic chemical or nuclear chain reactions the chain has a self-enforcing effect and after an initial cue the concentration of the system components are able to grow by themselves. Consumer chains include food chains but are more general and consumption of different commodities are also taken into account such as water, energy, raw materials, technology and information. An advanced consumer chain model considers both the limited amount of resources and the cooperation of consumers. Depending on the specific circumstances both properties play a role or one of them dominates. For example if the consumption rate is small and resources are plentiful their limited amount is not felt and it can be ignored in a realistic model. If consumers cooperate they will be able to utilize other constituents of the chain very efficiently with increasing concentration and some of the nonlinear terms in system may be superlinear.

In this respect, it is interesting to consider the work of Bettencourt and West [3] who collected extensive empirical data on typical activities in cities such as scientific publications or patents, GDP or the number of educational institutions but also crime, traffic congestion or certain diseases indicating that they grow at a superlinear rate. They established a universal growth rate which applies to most of the activities in major cities independent of geographic location or ethnicity of the population and cultural background. In our model we consider this situation: the limited amount of resources is not felt and consumers cooperative to utilize nutrients and other supplies very efficiently.

In biology consumers and suppliers are often called predator and prey. For background on predator-prey models we refer to [16]. Our system also serves as a model for a sequence of irreversible autocatalytic reactions in a container which is in contact with a well-stirred reservoir and similar models have been suggested before, see e.g. Chapter 8 of [25] and the references therein.

Although we do not consider genetic signalling pathways in this publication it is generally understood that their typical behaviour includes activator and inhibitor feedback loops between different components. Some work has been done on modelling their dynamics including stochastic approaches. On the other hand, in the vast majority of studies their spatial components are ignored. However, they are important for many settings, e.g. for the Wnt signaling pathway which describes interaction of cells and passing signals from the surface of the cell to its nucleus via a complex signaling pathway. It plays a role in embryonic development, cell differentiation and cell polarity generation. For further information we refer to the excellent review article [13]. A reaction-diffusion model for planar cell polarity is introduced and treated numerically in [1]. We plan to address these issues in future work by targeting our chain models more closely to the genetic signaling framework and including 
further typical ingredients stemming from genetic interaction such as typical lengthscales, mutual switch-on and switch-off mechanisms of genetic components, strength of interaction classified into ranges of growth and plateau levels, interaction of neighbouring cells resulting in spatio-temporal patterns at cellular or intracellular level, complex combinations of activator and inhibitor loops. In our example we just consider a chain of two activators which could be a first step in the modeling of complex signaling pathways and mathematical analysis of spatio-temporal structures for genetic signaling pathways which generally are represented by complex networks of multiple activators and inhibitors.

\section{A COOPERATIVE CONSUMER CHAIN MODEL}

We consider a reaction-diffusion system which serves as a cooperative consumer chain model. It considers the interaction of three components, one producer and two consumers, which supply each other in a sequence. It is an extension of the Schnakenberg model suggested in $[9,22]$ for which there is only one producer and one consumer. In this consumer chain model there is a middle component which plays a hybrid role: it acts both as consumer and producer. It is assumed that the producer diffuses much faster than the first consumer and the first consumer much faster than the second consumer.

This system can be written as follows:

$$
\left\{\begin{array}{l}
\tau \frac{\partial S}{\partial t}=D \Delta S+1-\frac{a_{1}}{\epsilon_{1}} S u_{1}^{2}, \quad x \in \Omega, t>0 \\
\tau_{1} \frac{\partial u_{1}}{\partial t}=\epsilon_{1}^{2} \Delta u_{1}-u_{1}+S u_{1}^{2}-a_{2} \frac{\epsilon_{1}}{\epsilon_{2}} u_{1} u_{2}^{2}, \quad x \in \Omega, t>0 \\
\frac{\partial u_{2}}{\partial t}=\epsilon_{2}^{2} \Delta u_{2}-u_{2}+u_{1} u_{2}^{2}, \quad x \in \Omega, t>0
\end{array}\right.
$$

where $S$ and $u_{i}$ denote the concentrations of producer and the two consumers, respectively. Here $0<\epsilon_{2}^{2} \ll \epsilon_{1}^{2} \ll 1$ and $0<D$ are three positive diffusion constants. There are two small parameters: The diffusion constant $\epsilon_{1}^{2}$ and the ratio of the two small diffusion constants $\frac{\epsilon_{2}^{2}}{\epsilon_{1}^{2}}$. These two small parameters will play an important role throughout the paper. We also set

$$
\epsilon=\max \left\{\epsilon_{1}, \frac{\epsilon_{2}}{\epsilon_{1}}\right\}
$$

and will consider the limit $\epsilon \rightarrow 0$ which means that both $\epsilon_{1} \rightarrow 0$ and $\frac{\epsilon_{2}}{\epsilon_{1}} \rightarrow 0$. The constants $a_{1}, a_{2}$ (positive) for the feed rates and $\tau, \tau_{1}$ (nonnegative) for the time relaxation constants will be treated as parameters and their choices will determine existence and stability properties of steady-state cluster solutions. Note that the overall supply rates $\frac{a_{1}}{\epsilon_{1}}$ and $a_{2} \frac{\epsilon_{1}}{\epsilon_{2}}$ are large, and they increase as the two small parameters decrease.

The system will be considered on the interval $\Omega=(-1,1)$ with Neumann boundary conditions for $t>0$ :

$$
\frac{d S}{d x}(-1, t)=\frac{d S}{d x}(1, t)=0, \quad \frac{d u_{1}}{d x}(-1, t)=\frac{d u_{1}}{d x}(1, t)=0, \quad \frac{d u_{2}}{d x}(-1, t)=\frac{d u_{2}}{d x}(1, t)=0 .
$$

The model (2.1) represents a consumer chain under the assumption that different social groups interact on vastly different scales, e.g. worldwide - national - regional, or national 
- regional - local. Typically the spatial scales of consumers are much smaller than those of the resources they use. For example major cities can only exist by importing most of their natural resources, e.g. materials, energy, food as well as consumer products through a complex logistic supply chain. Even within cities there are often certain areas such as central business districts or residential neighborhoods which on the one hand interact very efficiently within themselves but can only be sustained by receiving services and products from many other areas of the city on a sustained regular basis.

In cooperative and interconnected societies superlinear growth of consumption is frequently observed. For example Bettencourt and West showed empirically based on large data sets that in cities economic and socio-cultural activities grow superlinearly with size, whereby an increase in population of $100 \%$ results in growth of typical activities by approximately $115 \%$ [3]. For simplicity and easy mathematical treatability we have chosen quadratic nonlinearities in our model.

These observations provide a strong motivation to consider this consumer chain model with quadratic nonlinearities and diffusion coefficients having vastly different sizes. We consider the limit of two small diffusion constants which converge to zero at two different rates. This results in two different small spatial scales. Thus the model truly has the multiscale property.

We first prove the existence of cluster solutions in an interval for which the profile of the third component is that of the commonly observed spike in the Schnakenberg model. However, for the middle component a new cluster-type profile is observed which comes from the fact that it acts as producer and consumer simultaneously. Its profile is that of two partial spikes connected by a thin transition layer. In this profile different scales are involved: The spikes for the middle component are on the small scale, the spike for the last component on the very small scale, the width of the transition layer for the middle component is between the small and the very small scale. The first component is in leading order given by a Green's function and acts on the large scale. To our knowledge this type of cluster solution has never been studied before rigourously. It is shown that if the feed rates are small enough, more precisely if the combination $a_{1}^{2} a_{2}$ is below a certain threshold which has been characterized and computed explicitly, there exist two such cluster patterns which differ by their size.

We study the stability properties of this solution in terms of the system parameter using a rigorous approach to analyze the linearized operator around cluster solutions based on nonlocal eigenvalue problems and rigorous asymptotic analysis. The following result is established: If the time-relaxation constants are small enough, one cluster solutions is stable and and the other one is unstable. The instability comes through large eigenvalues of order $O(1)$. Further, there are small eigenvalues of order $o(1)$ which do not cause any instabilities.

The analysis uses some novel ideas:

(i) The consideration of the large eigenvalues leads to a new system of nonlocal eigenvalue problems which are coupled by an inhomogeneous Robin boundary condition. It is stated in (5.5), (5.6). Its stability properties system are determined by a rigorous approach.

(ii) The investigation of the small eigenvalues uses some new ideas to deal with the interaction of two small scales which is based on a suitable inner/outer expansion with rigorous 
error analysis. Remarkably, the order of the small eigenvalues is determined by the smallest diffusion constant $\epsilon_{2}^{2}$ and is intimately connected to the smallest length scale.

These results are generalizations of similar but much easier findings for the Schnakenberg model. Therefore, before stating our main results, let us briefly recall some previous studies for the Schnakenberg model or the related Gray-Scott model. In [12, 26] the existence and stability of spiky patterns on bounded intervals is established. In [34] similar results are shown for two-dimensional domains. In [2] it is shown how the degeneracy of the Turing bifurcation can be lifted using spatially varying diffusion coefficients. In [17, 18, 19] spikes are considered rigorously for the shadow system.

For the Gray-Scott model introduced in [10, 11], some of the results are the following. In $[5,6,7]$ the existence and stability of spiky patterns on the real line is proved. In [20, 21] a skeleton structure and separators for the Gray-Scott model are established.

Other "large" reaction diffusion systems (more than two components) with concentrated patterns include the hypercycle of Eigen and Schuster [8, 30, 32], and Meinhardt and Gierer's model of mutual exclusion and segmentation [15, 35].

The structure of this paper is as follows:

In Section 2, we state and explain the main theorems on existence and stability.

In Section 3 and Appendix A, we prove the main existence result, Theorem 3.1. In Section 3 , we compute the amplitudes of the spikes. In Appendix A, we give a rigorous existence proof.

In Section 4 and Appendix B, we prove the main stability result, Theorem 3.2. In Section 4, we derive a nonlocal eigenvalue problem (NLEP) and determine the stability of the $O(1)$ eigenvalues. In Appendix B, we study the stability of the $o(1)$ eigenvalues.

Finally, in Appendix C, we derive two Green's functions which are needed throughout the paper.

Throughout this paper, the letter $C$ will denote various generic constants which are independent of $\epsilon$, for $\epsilon$ sufficiently small. The notation $A \sim B$ means that $\lim _{\epsilon \rightarrow 0} \frac{A}{B}=1$; and $A=O(|B|)$ is defined as $|A| \leq C|B|$ for some $C>0 ; A=o(|B|)$ means that $\frac{|A|}{|B|} \rightarrow 0$.

\section{Main Results: Existence and Stability}

In this section we state the main results of this paper on existence and stability of cluster solutions. But we first need to introduce some notations and assumptions. We will construct stationary cluster solutions to (2.1), i.e. cluster solutions to the system

$$
\left\{\begin{array}{l}
D \Delta S+1-\frac{a_{1}}{\epsilon_{1}} S u_{1}^{2}=0, \quad x \in \Omega, \\
\epsilon_{1}^{2} \Delta u_{1}-u_{1}+S u_{1}^{2}-a_{2} \frac{\epsilon_{1}}{\epsilon_{2}} u_{1} u_{2}^{2}=0, \quad x \in \Omega, \\
\epsilon_{2}^{2} \Delta u_{2}-u_{2}+u_{1} u_{2}^{2}=0, \quad x \in \Omega
\end{array}\right.
$$

with the Neumann boundary conditions given in (2.3). The solutions of (3.1) will be even functions:

$$
S(|x|), \quad \text { where } S \in H_{N}^{2}(\Omega),
$$




$$
\begin{gathered}
u_{1}(|x|), \quad \text { where }\left(1-\chi\left(\frac{|x|}{\epsilon_{1} r_{\epsilon}}\right) u_{1} \in H_{N}^{2}\left(\Omega_{\epsilon_{1}}\right), \quad \chi\left(\frac{|x|}{\epsilon_{1} r_{\epsilon}}\right) u_{1} \in H_{N}^{2}\left(\Omega_{\epsilon_{2}}\right),\right. \\
u_{2}(|x|), \quad \text { where } u_{2} \in H_{N}^{1}\left(\Omega_{\epsilon_{2}}\right)
\end{gathered}
$$

with

$$
H_{N}^{2}(-1,1)=\left\{v \in H^{2}(-1,1): v^{\prime}(-1)=v^{\prime}(1)=0\right\}
$$

and

$$
\Omega_{\epsilon_{i}}=\left(-\frac{1}{\epsilon_{i}}, \frac{1}{\epsilon_{i}}\right), \quad i=1,2 .
$$

These solutions are bounded in their respective norms as $\epsilon=\max \left\{\epsilon_{1}, \frac{\epsilon_{2}}{\epsilon_{2}}\right\} \rightarrow 0$. Here $\chi$ is a smooth cutoff function which satisfies the following properties:

$$
\chi \in C_{0}^{\infty}(-1,1), \quad \chi(x)=1 \text { for }|x| \leq \frac{5}{8}, \quad \chi(x)=0 \text { for }|x| \geq \frac{3}{4}
$$

and

$$
r_{\epsilon}=10 \frac{\epsilon_{2}}{\epsilon_{1}} \log \frac{\epsilon_{1}}{\epsilon_{2}}
$$

Let $w$ be the unique solution of the problem

$$
\left\{\begin{array}{l}
w_{y y}-w+w^{2}=0, \quad w>0 \text { in } \mathbb{R}, \\
w(0)=\max _{y \in \mathbb{R}} w(y), \quad w(y) \rightarrow 0 \text { as }|y| \rightarrow+\infty
\end{array}\right.
$$

which is given by

$$
w(y)=\frac{3}{2 \cosh ^{2} \frac{y}{2}} .
$$

Before stating our main results, let us formally discuss how to derive the cluster solution by considering the interaction of the three scales. We set

$$
y_{i}=\frac{x}{\epsilon_{i}}, \quad i=1,2,
$$

and consider the limit $\epsilon \rightarrow 0$. From now on, we often drop the subscript $\epsilon$ if this does not cause confusion.

The third equation of (3.1) in leading order is given by

$$
\Delta_{y_{2}} u_{2}-u_{2}+u_{1}(0) u_{2}^{2} \sim 0
$$

and $u_{2}$ satisfies

$$
u_{2}\left(y_{2}\right) \sim \frac{1}{u_{1}(0)} w\left(y_{2}\right) .
$$

The middle equation of (3.1) in leading order is given by

$$
\Delta_{y_{1}} u_{1}-u_{1}+S(0) u_{1}^{2} \sim 0
$$

and $u_{1}$ satisfies

$$
u_{1}\left(y_{1}\right) \sim \frac{1}{S(0)} w\left(\left|y_{1}\right|-L_{0}\right) \quad \text { for } y_{1}>0
$$

where the constant $L_{0}>0$ has to be determined. Integrating the last term in the middle equation of (3.1) results in a jump condition at $y_{1}=0$ :

$$
u_{1, y_{1}}\left(0^{+}\right)-u_{1, y_{1}}\left(0^{-}\right) \sim a_{2} u_{1}(0) d_{1},
$$


where lower variables denote derivatives. The constant $d_{1}$ has to be worked out from the asymptotic behavior of $u_{2}$ given in (3.7). For $u_{1}$ we use the asymptotic behavior of $u_{1}$ given in (3.8). This implies our first solvability condition.

Finally, the first equation of (3.1) in leading order is given by

$$
D \Delta S+1-S(0) \delta_{0} d_{0} \sim 0
$$

where $\delta_{0}$ is the Dirac distribution. The constant $d_{0}$ has to be worked out using the asymptotic behavior of $u_{1}$ given in (3.8). Then $S$ can be computed using a Green's function which will be defined in (9.1). Here, to have a solution for $S$, the integral of the last two terms must vanish. This implies our second solvability condition.

Introducing the notation $S_{0}=S(0), z=\tanh \left(\frac{L_{0}}{2}\right)$, the two solvability conditions can be written as follows:

$$
\begin{aligned}
& S_{0}=a_{1}\left(6+9 z-3 z^{3}\right), \\
& S_{0}=\frac{\sqrt{3}}{2 \sqrt{a_{2}}} \sqrt{z}\left(1-z^{2}\right) .
\end{aligned}
$$

We will show that, if $a_{1}^{2} a_{2}$ is small enough, there are two solutions $\left(S_{0}, L_{0}\right)$ such that

$$
0<L_{0}^{s}<L_{0}^{m}<L_{0}^{l}
$$

where $L_{0}^{m} \approx 0.6380$. Otherwise there are no solutions $\left(S_{0}, L_{0}\right)$. (At the threshold value for $a_{1}^{2} a_{2}$ there is exactly one solution $\left(S_{0}, L_{0}\right)$.)

Using $S_{0}$ and $L_{0}$, the other properties of the cluster solution can now be worked out easily.

Actually, a finer analysis of the behavior of $u_{1}$ near zero is required on the $y_{2}$ scale. Basically, we obtain $u_{1}$ in that inner region by integrating the last term in the middle equation of (3.1) on the $y_{2}$ scale using the profile of $u_{2}$. The details will be given below (see the function $u_{1 a, \epsilon}$ which will be introduced in (4.8)).

The rigorous mathematical statement is given in the following main existence result.

Theorem 3.1. Assume that

$$
\epsilon=\max \left\{\epsilon_{1}, \frac{\epsilon_{2}}{\epsilon_{1}}\right\} \ll 1, \quad D=\text { const. }
$$

and

$$
a_{1}^{2} a_{2}<c_{0}
$$

where

$$
c_{0}=\max _{0<z<1} \frac{z(z-1)^{2}}{9(z-2)^{2}(z+1)^{2}} \approx 0.0025 .
$$

Then problem (3.1) admits two "cluster" solutions $\left(S_{\epsilon}^{s}, u_{1, \epsilon}^{s}, u_{2, \epsilon}^{s}\right)$ and $\left(S_{\epsilon}^{l}, u_{1, \epsilon}^{l}, u_{2, \epsilon}^{l}\right)$ with the following properties:

(1) all components are even functions.

(2) $S_{\epsilon}(0)$ satisfies

$$
S_{\epsilon}(0)=S_{0}+O\left(\epsilon \log \frac{1}{\epsilon}\right)
$$


(3)

$$
\begin{gathered}
u_{1, \epsilon}(x)=\xi_{1}^{\epsilon}\left[w\left(\frac{|x|}{\epsilon_{1}}-L_{0}-r_{\epsilon}\right)\left(1-\chi\left(\frac{|x|}{\epsilon_{1} r_{\epsilon}}\right)\right)\right. \\
\left.+\left(u_{1, \epsilon}(0)+\frac{\epsilon_{2}}{\epsilon_{1}} u_{1 a, \epsilon}\left(\frac{|x|}{\epsilon_{2}}\right)\right) \chi\left(\frac{|x|}{\epsilon_{1} r_{\epsilon}}\right)\right]+O\left(\epsilon \log \frac{1}{\epsilon}\right), \\
u_{2, \epsilon}(x)=\xi_{2}^{\epsilon} w\left(\frac{|x|}{\epsilon_{2}}\right)+O\left(\epsilon \log \frac{1}{\epsilon}\right)
\end{gathered}
$$

where $\chi$ is a smooth cutoff function given in (3.3),

$$
\xi_{1}^{\epsilon}=\frac{1}{S_{\epsilon}\left(\epsilon_{1} r_{\epsilon}\right)}=\frac{1}{S_{0}}+O\left(\epsilon_{1} r_{\epsilon}\right), \quad \xi_{2}^{\epsilon}=\frac{1}{u_{1, \epsilon}(0)}=\frac{1}{\xi_{1}^{\epsilon} w\left(-L_{0}\right)}+O\left(r_{\epsilon}\right)=\frac{S_{0}}{w\left(-L_{0}\right)}+O\left(r_{\epsilon}\right),
$$

$r_{\epsilon}$ has been defined in (3.4) and $w$ is the unique solution of (3.5), The estimates are in the sense of the norms given in (3.2). The function $u_{1 a, \epsilon}$ describing $u_{1, \epsilon}$ in the inner region will be introduced in (4.8).

(4) $L_{0}$ and $S_{0}$ are determined by solving the system (3.9), (3.10) which has two solutions $\left(S_{0}^{s}, L_{0}^{s}\right),\left(S_{0}^{l}, L_{0}^{l}\right)$, where

$$
0<L_{0}^{s}<L_{0}^{m}<L_{0}^{l}
$$

with $L_{0}^{m} \approx 0.6380$.

Finally, if $a_{1}^{2} a_{2}>c_{0}$, then for $\epsilon$ small enough there are no cluster solutions which satisfy (1) $-(4)$.

Theorem 3.1 will be proved in Section 3 and Appendix A.

\section{Remarks.}

1. Note that

$$
\epsilon_{2} \ll \epsilon_{1} r_{\epsilon} \ll \epsilon_{1},
$$

i.e. the scale of $\epsilon_{1} r_{\epsilon}$ is between the very small scale and the small scale.

2. If $L_{0}^{s}<L_{0}^{l}$ then, by varying $a_{1}$ and $a_{2}$, it is possible for the corresponding value of $S_{0}$ to satisfy either $S_{0}^{s}<S_{0}^{l}$ or $S_{0}^{l}<S_{0}^{s}$. This means that the cluster in the larger interval could have larger or smaller amplitude.

3. Expressed more precisely, (3.11) means that $\epsilon_{1}$ and $\frac{\epsilon_{2}}{\epsilon_{1}}$ are small enough; (3.12) means the following: for every $\delta_{0}>0$ there exists and $\epsilon_{0}>0$ such that for all $\epsilon_{1}, \epsilon_{2}$ which satisfy $0<\epsilon_{1}<\epsilon_{0}$ and $0<\frac{\epsilon_{2}}{\epsilon_{1}}<\epsilon_{0}$ we have $a_{1}^{2} a_{2}<c_{0}-\delta_{0}$. The assumption in the last sentence of the theorem is to be understood in the same way.

4. We remark that using spaces of even functions will make the existence proof easier since the single spike for $u_{2}$ must be located at the center and translations of it are automatically excluded.

The second main result of this paper concerns the stability properties of the cluster solutions constructed in Theorem 3.1 and can be stated as follows:

Theorem 3.2. Assume that (3.11) and (3.12) are satisfied.

Let $\left(S_{\epsilon}^{l}, u_{1, \epsilon}^{l}, u_{2, \epsilon}^{l}\right)$ and $\left(S_{\epsilon}^{s}, u_{1, \epsilon}^{s}, u_{2, \epsilon}^{s}\right)$ be the cluster solutions given in Theorem 3.1.

Then we have the following: 
(1) (Stability) Suppose that $0 \leq \tau<\tau_{0}$, and $0 \leq \tau_{1}<\tau_{1,0}$, where $\tau_{0}>0$ and $\tau_{1,0}>0$ are suitable constants which may be chosen independently of $\epsilon_{1}$ and $\frac{\epsilon_{2}}{\epsilon_{1}}$. Then $\left(S_{\epsilon}^{l}, u_{1, \epsilon}^{l}, u_{2, \epsilon}^{l}\right)$ is linearly stable.

(2) (Instability) The solution $\left(S_{\epsilon}^{s}, u_{1, \epsilon}^{s}, u_{2, \epsilon}^{s}\right)$ is linearly unstable for all $\tau \geq 0$ and $\tau_{1} \geq 0$.

Theorem 3.2 implies that, in agreement with the Schnakenberg model, the small cluster solutions with $L_{0} \sim L_{0}^{s}$ are always linearly unstable [28, 29]. The large solutions with $L_{0} \sim L_{0}^{l}$ can be linearly stable or unstable, depending on certain conditions for the parameters of the system (2.1). To elucidate this issue, we will rigorously investigate their stability behavior in detail. Theorem 3.2 will be proved in Section 4 and Appendix B.

\section{Existence I: Formal COMputation of the Amplitudes}

In this section and Appendix A, we will show the existence of cluster solutions to system (3.1) and prove Theorem 3.1. In this section we determine $S$ and $L_{0}$.

We choose the approximate solution to (3.1) as follows:

$$
\begin{gathered}
u_{1, \epsilon}(x)=\xi_{1}^{\epsilon}\left[w\left(\frac{|x|}{\epsilon_{1}}-L_{0}-r_{\epsilon}\right)\left(1-\chi\left(\frac{|x|}{\epsilon_{1} r_{\epsilon}}\right)\right) \chi(|x|)\right. \\
\left.+\left(u_{1, \epsilon}(0)+\frac{\epsilon_{2}}{\epsilon_{1}} u_{1 a, \epsilon}\left(\frac{|x|}{\epsilon_{2}}\right)\right) \chi\left(\frac{|x|}{\epsilon_{1} r_{\epsilon}}\right)\right] \\
u_{2, \epsilon}(x)=\xi_{2}^{\epsilon} w\left(\frac{|x|}{\epsilon_{2}}\right) \chi\left(\frac{|x|}{\epsilon_{1} r_{\epsilon}}\right)
\end{gathered}
$$

where $\xi_{i}, i=1,2$, are positive constants to be determined. They will follow from the solution $\left(S_{0}, L_{0}\right)$ computed in this section.

Substituting (3.16) into the last equation of (3.1) and using (3.5), we compute

$$
\xi_{2}^{\epsilon}=\frac{1}{u_{1, \epsilon}(0)} .
$$

Substituting (3.1) into the second equation of (3.1) and using (3.5), we get

$$
\xi_{1}^{\epsilon}=\frac{1}{S_{\epsilon}\left(\epsilon_{1} r_{\epsilon}\right)}=\frac{1}{S_{\epsilon}(0)}+O\left(\epsilon_{1} r_{\epsilon}\right) .
$$

Next we will derive the two solvability conditions which determine the limiting amplitude $S_{0}$ of the source and half of the middle spike distance $L_{0}$ (or equivalently, for $z:=\tanh \left(\frac{L_{0}}{2}\right)$ ) which have been stated in (3.9), (3.10). Then we will use these two conditions to determine $S_{0}$ and $L_{0}$.

We begin by substituting (4.1) with (4.2) and (4.3) in (3.1).

Integrating the first equation in (3.1), using the Neumann boundary condition and balancing the last two terms, we get

$$
\begin{gathered}
1=2 a_{1}\left(\frac{1}{S_{\epsilon}(0)} \int_{-L_{0}}^{\infty} w^{2}\left(y_{1}\right) d y_{1}+S_{\epsilon}(0) \int_{0}^{r_{\epsilon}}\left(u_{1, \epsilon}^{2}(0)+O\left(r_{\epsilon}\right)\right) d y_{1}\right)\left(1+O\left(\epsilon_{1}\right)\right) \\
=\frac{2 a_{1}}{S_{\epsilon}(0)}\left(\int_{-L_{0}}^{\infty} w^{2}\left(y_{1}\right) d y_{1}\right)\left(1+O\left(\epsilon \log \frac{1}{\epsilon}\right)\right)
\end{gathered}
$$


using

$$
S_{\epsilon}\left(\epsilon_{1} y_{1}\right)=S_{\epsilon}(0)+O\left(\epsilon_{1}\left|y_{1}\right|\right) \text {. }
$$

Denoting

$$
\rho(L):=\int_{0}^{L} w^{2}(y) d y=\frac{3}{2} \tanh \left(\frac{L}{2}\right)\left(3-\tanh ^{2}\left(\frac{L}{2}\right)\right),
$$

(see equation (2.3) in [36]), which implies as a special case

$$
\int_{-\infty}^{\infty} w^{2}(y) d y=6
$$

we can rewrite (4.4) as

$$
S_{\epsilon}(0)=a_{1}\left(6+9 z-3 z^{3}\right)+O\left(r_{\epsilon}\right) .
$$

Taking the limit $\epsilon \rightarrow 0$ implies our first condition for $S_{0}$ and $z$ given in (3.9):

$$
S_{0}=a_{1}\left(6+9 z-3 z^{3}\right) .
$$

Using (3.1) and (4.3), we get

$$
u_{1, \epsilon}\left(r_{\epsilon}\right)=\frac{1}{S_{\epsilon}(0)} w\left(-L_{0}\right)+O\left(\epsilon_{1} r_{\epsilon}\right)
$$

which implies

$$
u_{1, \epsilon}(0)=u_{1, \epsilon}\left(r_{\epsilon}\right)+O\left(r_{\epsilon}\right)=\frac{1}{S_{\epsilon}(0)} w\left(-L_{0}\right)+O\left(r_{\epsilon}\right) .
$$

Using (3.16) and (4.2), we derive

$$
u_{2, \epsilon}(0)=\frac{1}{u_{1, \epsilon}(0)} w(0)=\frac{S_{\epsilon}(0)}{w\left(-L_{0}\right)} w(0)+O\left(r_{\epsilon}\right),
$$

where $S_{\epsilon}(0)$ and $L_{0}$ are unknown constants to be determined.

On the $\epsilon_{2}$ scale, we compute from the second equation of (3.1) for $\left|y_{2}\right| \leq \frac{\epsilon_{1}}{\epsilon_{2}} r_{\epsilon}=\log \frac{\epsilon_{1}}{\epsilon_{2}}$

$$
\begin{gathered}
u_{1 a, \epsilon}^{\prime \prime}\left(y_{2}\right)=a_{2} u_{1, \epsilon}(0) u_{2, \epsilon}^{2}\left(y_{2}\right)\left(1+O\left(r_{\epsilon}\right)\right) \\
=a_{2} \frac{1}{u_{1, \epsilon}(0)} w^{2}\left(y_{2}\right)\left(1+O\left(r_{\epsilon}\right)\right) .
\end{gathered}
$$

Integrating gives

$$
u_{1 a, \epsilon}^{\prime}\left(y_{2}\right)=a_{2} \frac{1}{u_{1, \epsilon}(0)} \rho\left(y_{2}\right)\left(1+O\left(r_{\epsilon}\right)\right)
$$

and

$$
u_{1 a, \epsilon}\left(y_{2}\right)=c+a_{2} \frac{1}{u_{1, \epsilon}(0)} \int_{0}^{y_{2}} \rho(s) d s\left(1+O\left(r_{\epsilon}\right)\right) .
$$

This implies

$$
\lim _{y_{2} \rightarrow \pm \infty} u_{1 a, \epsilon}^{\prime}\left(y_{2}\right)=a_{2} \frac{1}{u_{1, \epsilon}(0)} \lim _{y_{2} \rightarrow \pm \infty} \rho\left(y_{2}\right)\left(1+O\left(r_{\epsilon}\right)\right)=a_{2} \frac{1}{u_{1}(0)}( \pm 3)\left(1+O\left(r_{\epsilon}\right)\right) .
$$

Considering even functions, matching

$$
\lim _{y_{2} \rightarrow \pm \infty} u_{1 a, \epsilon}^{\prime}\left(y_{2}\right)=u_{1, \epsilon}^{\prime}\left( \pm r_{\epsilon}\right)
$$


and using (4.6), (4.9), we get

$$
2 u_{1, \epsilon}^{\prime}\left(r_{\epsilon}\right)=a_{2} \frac{6 S_{\epsilon}(0)}{w\left(-L_{0}\right)}+O\left(r_{\epsilon}\right)
$$

From (3.6), we compute

and

$$
w\left(-L_{0}\right)=\frac{3}{2}\left(1-z^{2}\right)
$$

$$
\begin{gathered}
2 u_{1, \epsilon}^{\prime}\left(r_{\epsilon}\right)=\frac{2}{S_{\epsilon}(0)}\left(w^{\prime}\left(-L_{0}\right)\right)(1+O(\epsilon)) \\
=\frac{2}{S_{0}} \tanh \left(\frac{L_{0}}{2}\right) w\left(-L_{0}\right)(1+O(\epsilon)) \\
=\frac{3 z\left(1-z^{2}\right)}{S_{0}}
\end{gathered}
$$

Taking limits in (4.11) implies our second condition for $S_{0}$ and $z$ given in (3.10):

$$
S_{0}=\frac{\sqrt{3}}{2 \sqrt{a_{2}}} \sqrt{z}\left(1-z^{2}\right) .
$$

Next we determine $S_{0}$ and $z$ from (3.9) and (3.10). Equating (3.9) and (3.10) gives

$$
\frac{\sqrt{z}(z-1)}{3(z-2)(z+1)}=\frac{2 a_{1} \sqrt{a_{2}}}{\sqrt{3}} .
$$

Elementary computations show that the function on the l.h.s. of (4.12) vanishes for $z=0$ or $z=1$ and it has a unique maximum for $z$ in the interval $(0,1)$. This maximum is reached for $z^{m} \approx 0.30851$ which corresponds to $L_{0}^{m} \approx 0.6380$. The value of the maximum of l.h.s. is $\approx 0.0578$.

Now in Figure 1 we plot the l.h.s. of (4.12) with $z=\tanh \left(\frac{L_{0}}{2}\right)$ versus $L_{0}=$.

Figure 1. This graph shows the l.h.s. of (4.12) versus $L_{0}$. The maximum of l.h.s. of (4.12) is reached for $L_{0}^{m} \approx 0.6380$, the value reached is $\approx 0.0578$.

This implies that under the condition

$$
a_{1}^{2} a_{2}<c_{0}, \quad \text { where } c_{0} \approx 0.0025,
$$


there are two solutions $\left(S_{0}, L_{0}\right)$ for which $L_{0}$ satisfies

$$
0<L_{0}^{s}<L_{0}^{m}<L_{0}^{l}
$$

If

$$
a_{1}^{2} a_{2}>c_{0}
$$

there are no such solutions. Trivially, the large $z$ corresponds to the large $L_{0}$.

This result can be interpreted as follows: To have this type of cluster solution, the feed rates for both $a_{1}$ and $a_{2}$ must be small enough. Otherwise the producers $S$ and $u_{1}$ will not be able to sustain the consumers $u_{1}$ and $u_{2}$, respectively. Instead, among others, the following behaviors are possible:

(i) The component $u_{2}$ will die out and a spike for the Schnakenberg model remains which involves only the components $S$ and $u_{1}$ with $u_{2}=0$.

(ii) The component $u_{2}$ will die out and $u_{1}, S$ will both approach positive constants. It can easily be seen that

$$
u_{1}=\frac{\epsilon_{1}}{a_{1}}, \quad S=\frac{a_{1}}{\epsilon_{1}} .
$$

(iii) The components approach the positive homogeneous steady state

$$
S=\frac{\epsilon_{1}}{a_{1} u_{1}^{2}}, \quad u_{1}^{2}-\frac{\epsilon_{1}}{a_{1}} u_{1}+a_{2} \frac{\epsilon_{1}}{\epsilon_{2}}=0, \quad u_{2}=\frac{1}{u_{1}} .
$$

Figure 2 shows the spatial profiles of the steady states $S, u_{1}, u_{2}$ for parameters $D=$ $10, \epsilon_{1}^{2}=10^{-4}, \epsilon_{2}^{2}=10^{-8}, \frac{a_{1}}{\epsilon}=10, a_{2} \frac{\epsilon_{1}}{\epsilon_{2}}=1$. Note that the small space variables are $y_{1}=$ $\epsilon_{1} x=10^{-2} x$ (scale of the two spikes for $u_{1}$ ) and $y_{2}=\epsilon_{2} x=10^{-4} x$ (scale of the spike for $u_{2}$ ).

Figure 2a. The spatial profiles of the steady states $S, u_{1}, u_{2}$ for parameters $D=10, \epsilon_{1}^{2}=$ $10^{-4}, \epsilon_{2}^{2}=10^{-8}, \frac{a_{1}}{\epsilon}=10, a_{2} \frac{\epsilon_{1}}{\epsilon_{2}}=1$. 
Figure 2b. Same as Figure 2a, but zoomed in for the spatial scale.

In the next section we analyze the stability properties of the cluster steady states.

\section{Stability I: Derivation, Rigorous Deduction and Analysis of a NLEP}

We linearize (2.1) around the cluster solution $S_{\epsilon}+\psi_{\epsilon} e^{\lambda t}, u_{\epsilon, i}+\phi_{\epsilon, i} e^{\lambda t}, i=1,2$, and study the eigenvalue problem of the resulting linearized operator:

$$
\mathcal{L}_{\epsilon}\left(\begin{array}{c}
\psi_{\epsilon} \\
\phi_{1, \epsilon} \\
\phi_{2, \epsilon}
\end{array}\right)=\left(\begin{array}{c}
\tau \lambda_{\epsilon} \psi_{\epsilon} \\
\tau_{1} \lambda_{\epsilon} \phi_{1, \epsilon} \\
\lambda_{\epsilon} \phi_{2, \epsilon}
\end{array}\right),
$$

where $\mathcal{L}_{\epsilon}$ denotes the linearized operator.

We assume that the domain of the operator $\mathcal{L}_{\epsilon}$ is $H_{N}^{2}(\Omega) \times H_{N}^{2}\left(\Omega_{\epsilon_{1}}\right) \times H_{N}^{2}\left(\Omega_{\epsilon_{2}}\right)$ and that the eigenvalue satisfies $\lambda_{\epsilon} \in \mathbb{C}$, the set of complex numbers.

We say that a cluster solution is linearly stable if the spectrum $\sigma\left(\mathcal{L}_{\epsilon}\right)$ of $\mathcal{L}_{\epsilon}$ lies in a left half plane $\left\{\lambda \in \mathbb{C}: \operatorname{Re}(\lambda) \leq-c_{0}\right\}$ for some $c_{0}>0$. A cluster solution is called linearly unstable if there exists an eigenvalue $\lambda_{\epsilon}$ of $\mathcal{L}_{\epsilon}$ with $\operatorname{Re}\left(\lambda_{\epsilon}\right)>0$.

Next we write down $\mathcal{L}_{\epsilon}$ explicitly. First we express $\psi_{\epsilon}=T^{\prime}\left[\phi_{1, \epsilon}\right]$ using the Green's function $G_{D, \tau \lambda_{\epsilon}}$ defined in (9.7), where $\psi_{\epsilon}=T^{\prime}\left[\phi_{1, \epsilon}\right]$ is the unique solution of the equation

$$
D \Delta \psi_{\epsilon}-\frac{a_{1}}{\epsilon_{1}}\left(\psi_{\epsilon} u_{1, \epsilon}^{2}+2 S_{\epsilon} u_{1, \epsilon} \phi_{1, \epsilon}\right)=\tau \lambda_{\epsilon} \psi_{\epsilon} \quad x \in \Omega .
$$

Note that $\psi_{\epsilon}$ depends on $\phi_{1, \epsilon}$ but not on $\phi_{2, \epsilon}$. 
Then we can rewrite (5.1) as follows:

$$
\left\{\begin{array}{l}
\epsilon_{1}^{2} \Delta \phi_{1, \epsilon}-\phi_{1, \epsilon}+2 S_{\epsilon} u_{1, \epsilon} \phi_{1, \epsilon}+T^{\prime}\left[\phi_{1, \epsilon}\right] u_{1, \epsilon}^{2}-a_{2} \frac{\epsilon_{1}}{\epsilon_{2}} \phi_{1, \epsilon} u_{2, \epsilon}^{2}-2 a_{2} \frac{\epsilon_{1}}{\epsilon_{2}} u_{1, \epsilon} u_{2, \epsilon} \phi_{2, \epsilon}=\tau_{1} \lambda_{\epsilon} \phi_{1, \epsilon}, \\
\epsilon_{2}^{2} \Delta \phi_{2, \epsilon}-\phi_{2, \epsilon}+\phi_{1, \epsilon} u_{2, \epsilon}^{2}+2 u_{1, \epsilon} u_{2, \epsilon} \phi_{2, \epsilon}=\lambda_{\epsilon} \phi_{2, \epsilon} .
\end{array}\right.
$$

We decompose

$$
\phi_{1, \epsilon}\left(y_{1}\right)=\phi_{1 a, \epsilon}\left(\left|y_{1}\right|-L_{0}\right)\left(1-\chi\left(\frac{\left|y_{1}\right|}{r_{\epsilon}}\right)\right)+\frac{\epsilon_{2}}{\epsilon_{1}} \phi_{1 b, \epsilon} \chi\left(\frac{y_{1}}{r_{\epsilon}}\right)
$$

and assume that

$$
\| \mid\left(\phi_{1, \epsilon}, \phi_{2, \epsilon}\left\|\left.\right|_{L^{2}} ^{2}=\right\| \chi \phi_{1}\left\|_{L^{2}\left(\Omega_{\epsilon_{2}}\right)}^{2}+\right\|(1-\chi) \phi_{1}\left\|_{L^{2}\left(\Omega_{\epsilon_{1}}\right)}^{2}+\right\| \phi_{2} \|_{L^{2}\left(\Omega_{\epsilon_{2}}\right)}^{2}=1 .\right.
$$

Then, arguing as in the proof of Proposition 7.1 below, this sequence has a converging subsequence. We derive an eigenvalue problem for the limit. (Since we consider even eigenfunctions, it is enough to restrict our attention to the positive real axis.)

Now we first derive the limiting eigenvalue problem for the limit $\left(\phi_{1}, \phi_{2}\right)$ as $\epsilon \rightarrow 0$. Second we reduce it to a NLEP for $\phi_{2}$ only. In these computation we set $\tau=\tau_{1}=0$. At the end of the section we will show that considering small $\tau \geq 0$ and small $\tau_{1} \geq 0$ will only introduce a small perturbation.

For $\tau=0$, integrating the first equation in (5.3) gives

$$
\int_{\mathbb{R}^{+}} 2 S_{\epsilon} u_{1, \epsilon} \phi_{1, \epsilon} d x+\int_{\mathbb{R}^{+}} \psi_{\epsilon} u_{1, \epsilon}^{2} d x=0
$$

Taking the limit $\epsilon \rightarrow 0$ gives

$$
\frac{\psi(0)}{S_{0}^{2}(0)}=-\frac{2 \int_{-L_{0}}^{\infty} w \phi_{1} d y}{\int_{-L_{0}}^{\infty} w^{2} d y}
$$

Using (5.4), then the first equation in (5.3), after taking the limit $\epsilon \rightarrow 0$, leads to

$$
\Delta_{y_{1}} \phi_{1}-\phi_{1}+2 w \phi_{1}-\frac{2 \int_{-L_{0}}^{\infty} w \phi_{1}}{\int_{-L_{0}}^{\infty} w^{2}} w^{2}=0, \quad y>-L_{0} .
$$

The jump condition at $y=0$ translates into the boundary condition

$$
\phi_{1}^{\prime}\left(-L_{0}\right)-c \phi_{1}\left(-L_{0}\right)-c\left(u_{1}(0)\right)^{2} \frac{2 \int_{\mathbb{R}} w \phi_{2}}{\int_{\mathbb{R}} w^{2}}=0,
$$

where

$$
c=a_{2} \frac{S_{0}^{2}(0)}{w^{2}\left(-L_{0}\right)} 3 .
$$

Note that this is an inhomogeneous boundary condition of Robin type. Here we have used that

$$
u_{1}(0)=\frac{w\left(-L_{0}\right)}{S_{0}(0)}, \quad u_{2}(0)=\frac{1}{u_{1}(0)} .
$$

Similarly, the second equation in (5.3), after taking the limit $\epsilon \rightarrow 0$, leads to the eigenvalue problem

$$
\Delta_{y_{2}} \phi_{2}-\phi_{2}+2 w \phi_{2}+\frac{\phi_{1}\left(-L_{0}\right)}{\left(u_{1}(0)\right)^{2}} w^{2}=\lambda \phi_{2}, \quad y>0
$$


subject to the boundary condition

$$
\phi_{2}^{\prime}(0)=0
$$

For later use, we compute

$$
c=a_{2} \frac{3}{4 a_{2}} z\left(1-z^{2}\right)^{2} \frac{1}{\frac{9}{4}\left(1-z^{2}\right)^{2}} 3=z .
$$

We summarize this result as follows: Taking the limit $\epsilon \rightarrow 0$ in (5.3), we get

$$
\left\{\begin{array}{c}
\Delta_{y_{1}} \phi_{1}-\phi_{1}+2 w \phi_{1}-\frac{2 \int_{-L_{0}}^{\infty} w \phi_{1}}{\int_{-L_{0}}^{\infty} w^{2}} w^{2}=0, \quad y_{1}>-L_{0}, \\
\Delta_{y_{2}} \phi_{2}-\phi_{2}+2 w \phi_{2}+\frac{\phi_{1}\left(-L_{0}\right)}{\left(u_{1}(0)\right)^{2}} w^{2}=\lambda \phi_{2}, \quad y_{2} \in \mathbb{R},
\end{array}\right.
$$

with the boundary conditions

$$
\phi_{1}^{\prime}\left(-L_{0}\right)-c \phi_{1}\left(-L_{0}\right)-c\left(u_{1}(0)\right)^{2} \frac{2 \int_{\mathbb{R}} w \phi_{2}}{\int_{\mathbb{R}} w^{2}}=0, \quad \phi_{2}^{\prime}(0)=0 .
$$

Although the derivations given above are formal, we can rigorously prove the following separation of eigenvalues.

Theorem 5.1. Let $\lambda_{\epsilon}$ be an eigenvalue of (5.3) for which $\operatorname{Re}\left(\lambda_{\epsilon}\right)>-a_{0}$ with some suitable constant $a_{0}$ independent of $\epsilon$.

(1) Suppose that (for suitable sequences $\epsilon_{n} \rightarrow 0$ ) we have $\lambda_{\epsilon_{n}} \rightarrow \lambda_{0} \neq 0$. Then $\lambda_{0}$ is an eigenvalue of NLEP (5.10) with boundary condition (5.11).

(2) Let $\lambda_{0} \neq 0$ be an eigenvalue of NLEP (5.10) with boundary condition (5.11). Then, for $\epsilon$ sufficiently small, there is an eigenvalue $\lambda_{\epsilon}$ of (5.3) with $\lambda_{\epsilon} \rightarrow \lambda_{0}$ as $\epsilon \rightarrow 0$.

Remark: From Theorem 5.1 we see rigorously that the eigenvalue problem (5.3) is reduced to the study of the NLEP (5.10) with boundary conditions (5.11).

Now we prove Theorem 5.1.

\section{Proof of Theorem 5.1:}

Part (1) follows by an asymptotic analysis combined with passing to the limit as $\epsilon \rightarrow 0$ which is similar to the proof of Proposition 7.1 given below.

Part (2) follows from a compactness argument by Dancer introduced in Section 2 of [4]. It was applied in ([33]) to a related situation, therefore we omit the details.

The stability or instability of the large eigenvalues follows from the following results:

Theorem 5.2. [27]: Consider the following nonlocal eigenvalue problem

$$
\phi^{\prime \prime}-\phi+2 w \phi-\gamma \frac{\int_{\mathbb{R}} w \phi}{\int_{\mathbb{R}} w^{2}} w^{2}=\alpha \phi .
$$

(1) If $\gamma<1$, then there is a positive eigenvalue to (5.12).

(2) If $\gamma>1$, then for any nonzero eigenvalue $\alpha$ of (5.12), we have

$$
\operatorname{Re}(\alpha) \leq-c_{0}<0 .
$$

(3) If $\gamma \neq 1$ and $\alpha=0$, then $\phi=c_{0} w^{\prime}$ for some constant $c_{0}$. 
In our applications to the case when $\tau>0$ or $\tau_{1}>0$, we need to handle the situation when the coefficient $\gamma$ is a complex function of $\tau \alpha$. Let us suppose that

$$
\gamma(0) \in \mathbb{R}, \quad|\gamma(\tau \alpha)| \leq C \text { for } \alpha_{R} \geq 0, \tau \geq 0,
$$

where $C$ is a generic constant independent of $\tau, \alpha$.

Now we have

Theorem 5.3. (Theorem 3.2 of [33].)

Consider the following nonlocal eigenvalue problem

$$
\phi^{\prime \prime}-\phi+2 w \phi-\gamma(\tau \alpha) \frac{\int_{\mathbb{R}} w \phi}{\int_{\mathbb{R}} w^{2}} w^{2}=\alpha \phi,
$$

where $\gamma(\tau \alpha)$ satisfies (5.13). Then there is a small number $\tau_{0}>0$ such that for $\tau<\tau_{0}$,

(1) if $\gamma(0)<1$, then there is a positive eigenvalue to (5.12);

(2) if $\gamma(0)>1$, then for any nonzero eigenvalue $\alpha$ of (5.14), we have

$$
\operatorname{Re}(\alpha) \leq-c_{0}<0 .
$$

Now we consider the NLEP (5.10) and show the following result:

Lemma 5.1. The nonlocal eigenvalue problem (5.10) with boundary conditions (5.11) is stable for $L_{0}>L_{0}^{m}$ and unstable for $L_{0}<L_{0}^{m}$. For $L_{0} \neq L_{0}^{m}$ it does not have zero eigenvalue.

Proof of Lemma 5.1: Using Lemma 2.1 of [14] together with the Fredholm alternative, it follows that there is a unique solution $\phi_{1}$ of the problem (5.5) with boundary condition (5.6). We now compute this solution. It is easy to see that

$$
\phi_{1}(y)=\alpha w(y)+\beta w^{\prime}(y), \quad y>-L_{0},
$$

for some real constants $\alpha$ and $\beta$.

Plugging the ansatz (5.15) into (5.5), we compute

$$
\begin{gathered}
\alpha \underbrace{\left(w^{\prime \prime}-w+2 w^{2}\right)}_{=w^{2}}-\alpha \frac{2 \int_{-L_{0}}^{\infty} w^{2}}{\int_{-L_{0}}^{\infty} w^{2}} w^{2} \\
+\beta \underbrace{\left(w^{\prime \prime \prime}-w^{\prime}+2 w w^{\prime}\right)}_{=0}-\beta \frac{2 \int_{-L_{0}}^{\infty} w w^{\prime}}{\int_{-L_{0}}^{\infty} w^{2}} w^{2}=0, \quad y>-L_{0} .
\end{gathered}
$$

This implies

$$
\alpha-2 \alpha-2 \beta \frac{\int_{-L_{0}}^{\infty} w w^{\prime}}{\int_{-L_{0}}^{\infty} w^{2}}=0
$$

and finally

$$
\alpha=\beta \frac{w^{2}\left(-L_{0}\right)}{\rho\left(L_{0}\right)+3}
$$

since

$$
\int_{-L_{0}}^{\infty} w w^{\prime}=-\frac{1}{2} w^{2}\left(-L_{0}\right) .
$$


Using the boundary condition (5.6), we derive

$$
\alpha w^{\prime}\left(-L_{0}\right)+\beta w^{\prime \prime}\left(-L_{0}\right)-c\left(\alpha w\left(-L_{0}\right)+\beta w^{\prime}\left(-L_{0}\right)\right)-c u_{1}(0) \frac{2 \int_{\mathbb{R}} w \phi_{2}}{\int_{\mathbb{R}} w^{2}}=0 .
$$

Elementary computations give

$$
\begin{gathered}
\quad \frac{\beta}{u_{1}^{2}(0)}=\frac{c}{w^{\prime \prime}\left(-L_{0}\right)-c w^{\prime}\left(-L_{0}\right)} \frac{2 \int w \phi_{2}}{\int w^{2}} \\
=\frac{c}{-c w^{\prime}\left(-L_{0}\right)+w\left(-L_{0}\right)-w^{2}\left(-L_{0}\right)} \frac{2 \int w \phi_{2}}{\int w^{2}} .
\end{gathered}
$$

Then by Theorem 5.2 the NLEP (5.10) is stable if

$$
\gamma=-\frac{\phi_{1}\left(-L_{0}\right)}{u_{1}^{2}(0)}\left(\frac{\int w \phi_{2}}{\int w^{2}}\right)^{-1}>1
$$

We compute, using (5.15),

Using

$$
\begin{gathered}
\gamma=-\frac{\phi_{1}\left(-L_{0}\right)}{u_{1}^{2}(0)}\left(\frac{\int w \phi_{2}}{\int w^{2}}\right)^{-1}=-\frac{\beta}{u_{1}^{2}(0)}\left(\frac{\alpha}{\beta} w\left(-L_{0}\right)+w^{\prime}\left(-L_{0}\right)\right)\left(\frac{\int w \phi_{2}}{\int w^{2}}\right)^{-1} \\
=-\frac{\beta}{u_{1}^{2}(0)}\left(\frac{w^{3}\left(-L_{0}\right)}{\rho\left(L_{0}\right)+3}+w^{\prime}\left(-L_{0}\right)\right)\left(\frac{\int w \phi_{2}}{\int w^{2}}\right)^{-1} \\
=\frac{c\left(\frac{w^{3}\left(-L_{0}\right)}{\rho\left(L_{0}\right)+3}+w^{\prime}\left(-L_{0}\right)\right)}{c w^{\prime}\left(-L_{0}\right)-w\left(-L_{0}\right)+w^{2}\left(-L_{0}\right)} 2 .
\end{gathered}
$$

$$
z=\tanh \left(\frac{L_{0}}{2}\right) \quad\left(\text { and } c=z, w^{\prime}\left(-L_{0}\right)=z w, w\left(-L_{0}\right)=\frac{3}{2}\left(1-z^{2}\right)\right),
$$

the condition $\gamma>1$ can be rewritten as

$$
\frac{z \frac{\frac{27}{8}\left(1-z^{2}\right)^{3}}{3+\frac{9}{2} z-\frac{3}{2} z^{3}}+\frac{3}{2} z^{2}\left(1-z^{2}\right)}{\frac{3}{2} z^{2}\left(1-z^{2}\right)-\frac{3}{2}\left(1-z^{2}\right)+\frac{9}{4}\left(1-z^{2}\right)^{2}}>\frac{1}{2} .
$$

This is equivalent to

and to

$$
\frac{z \frac{27}{8}\left(1-z^{2}\right)^{3}}{3+\frac{9}{2} z-\frac{3}{2} z^{3}}>\frac{3}{8}\left(1-5 z^{2}\right)\left(1-z^{2}\right)
$$

$$
6 z(z-1)^{2}>\left(5 z^{2}-1\right)(z-2)
$$

We compare this result with Figure 1. Taking the derivative of 1.h.s. of (4.12) w.r.t. $z$, we compute

$$
\begin{gathered}
\frac{d}{d z} \frac{\sqrt{z}(z-1)}{3(z-2)(z+1)} \\
=\frac{\left(5 z^{2}-1\right)(z-2)-6 z(z-1)^{2}}{6 \sqrt{z}(z-2)^{2}(z+1)^{2}}<0
\end{gathered}
$$

which is equivalent to the condition (5.17). This means that we have linearized stability for the large eigenvalues at the decreasing part of the graph in Figure 1 for which $L_{0}$ and $z$ 
are large. On the other hand, we have linearized instability for the large eigenvalues at the increasing solution branch, for which $L_{0}$ and $z$ are small.

Finally, we continue to consider the stability problem for the linearized operator by extending our approach to the case $\tau \geq 0$ small and $\tau_{1} \geq 0$ small.

First note that $\psi$ is continuous in $\tau \lambda$ which follows by using Green's function to solve (5.2) for $\psi_{\epsilon}=T^{\prime}\left[\phi_{1, \epsilon}\right]$.

Second, using (5.15) in a perturbed version of (5.5), we derive that now

$$
\phi_{1}=\alpha w+\beta w^{\prime}+O\left(\left(\tau+\tau_{1}\right)|\lambda|\right)
$$

with the same values for $\alpha$ and $\beta$ as before.

Third, integrating the second equation in (5.3) near zero, taking the limit $\epsilon \rightarrow 0$ and using the perturbed boundary condition (5.6), now we get

$$
\begin{gathered}
\gamma=-\frac{\phi_{1}\left(-L_{0}\right)}{u_{1}(0)}\left(\frac{\int w \phi_{2}}{\int w^{2}}\right)^{-1}+O\left(\left(\tau+\tau_{1}\right)|\lambda|\right)= \\
=\frac{c\left(\frac{w^{3}\left(-L_{0}\right)}{\rho\left(L_{0}\right)+3}+w^{\prime}\left(-L_{0}\right)\right)}{c w^{\prime}\left(-L_{0}\right)-w\left(-L_{0}\right)+w^{2}\left(-L_{0}\right)} 2+O\left(\left(\tau+\tau_{1}\right)|\lambda|\right) .
\end{gathered}
$$

Finally, multiplying the eigenvalue problem (5.3) by the eigenfunction and using quadratic forms, it can be shown that $|\lambda|$ is bounded for $\tau$ and $\tau_{1}$ small enough. This argument is given in detail in [31] and we refer to that work for further information. This implies that small values of $\tau \geq 0$ and $\tau_{1} \geq 0$ introduce only small perturbations to the eigenvalue problem for large eigenvalues $\lambda_{\epsilon}$ of order $O(1)$ and Theorem 3.2 continues to hold in this case.

For the existence proof in Appendix A we will need to know that the adjoint operator $\mathcal{L}_{\epsilon}^{*}$ to the linear operator $\mathcal{L}_{\epsilon}$ is invertible. This is the issue of our next result.

Expressing $\mathcal{L}_{\epsilon}^{*}$ explicitly, we can rewrite the adjoint eigenvalue problem as follows:

$$
\left\{\begin{array}{l}
D \Delta \psi_{\epsilon}+\frac{1}{\epsilon_{1}}\left(\phi_{1, \epsilon}-a_{1} \psi_{\epsilon}\right) u_{1, \epsilon}^{2}=\tau_{1} \lambda_{\epsilon} \psi_{\epsilon} \\
\epsilon_{1}^{2} \Delta \phi_{1, \epsilon}-\phi_{1, \epsilon}+2 S_{\epsilon} u_{1, \epsilon}\left(\phi_{1, \epsilon}-a_{1} \psi_{\epsilon}\right)+\frac{\epsilon_{1}}{\epsilon_{2}}\left(\phi_{2, \epsilon}-a_{2} \phi_{1, \epsilon}\right) u_{2, \epsilon}^{2}=\tau_{1} \lambda_{\epsilon} \phi_{1, \epsilon}, \\
\epsilon_{2}^{2} \Delta \phi_{2, \epsilon}-\phi_{2, \epsilon}+2 u_{1, \epsilon} u_{2, \epsilon}\left(\phi_{2, \epsilon}-a_{2} \phi_{1, \epsilon}\right)=\lambda_{\epsilon} \phi_{2, \epsilon} .
\end{array}\right.
$$

Taking the limit $\epsilon \rightarrow 0$, we get the following limiting adjoint eigenvalue problem

$$
\left\{\begin{array}{l}
\Delta_{y_{1}} \phi_{1}-\phi_{1}+2 w \phi_{1}-2 \frac{\int_{-L_{0}}^{\infty} w^{2} \phi_{1}}{\int_{-L_{0}}^{\infty} w^{2}} w=\tau_{1} \lambda_{\epsilon} \phi_{1} \\
\Delta_{y_{2}} \phi_{2}-\phi_{2}+2 w \phi_{2}-2 a_{2} \phi_{1}(0) w=\lambda_{\epsilon} \phi_{2} \\
\phi_{1}^{\prime}\left(-L_{0}\right)-c \phi_{1}\left(-L_{0}\right)+\frac{c}{a_{2}} u_{1}(0) \frac{\int_{\mathbb{R}} w^{2} \phi_{2}}{\int_{\mathbb{R}} w^{2}}=0 \\
\phi_{2}^{\prime}(0)=0
\end{array}\right.
$$

We are now going to show that this limit of the adjoint operator has only the trivial kernel and prove the following lemma: 
Lemma 5.2. The limiting adjoint eigenvalue problem given in (5.19) has only the trivial kernel.

\section{Proof of Lemma 5.2:}

We introduce the notation $\mathcal{L} \phi:=\Delta \phi-\phi+2 w \phi, y>-L_{0}, \quad \phi^{\prime}\left(-L_{0}\right)-c \phi\left(-L_{0}\right)=0$.

It is easy to see that

$$
\phi_{1}(y)=\alpha\left(\mathcal{L}^{-1} w\right)(y)+\beta w^{\prime}(y), \quad y>-L_{0},
$$

for some real constants $\alpha$ and $\beta$.

From Lemma 2.2 in [14], we get

$$
\mathcal{L}^{-1} w(y)=w+\frac{1}{2}\left(y+L_{0}\right) w^{\prime}+\frac{z}{1-z^{2}} w^{\prime} .
$$

Plugging (5.20) into (5.19), we compute

$$
\begin{gathered}
\alpha \underbrace{\mathcal{L}\left(\mathcal{L}^{-1} w\right)}_{=w}-\alpha \frac{2 \int_{-L_{0}}^{\infty} w^{2}\left(\mathcal{L}^{-1} w\right) d y}{\int_{-L_{0}}^{\infty} w^{2} d y} w \\
+\beta \underbrace{\mathcal{L} w^{\prime}}_{=0}-\beta \frac{2 \int_{-L_{0}}^{\infty} w^{2} w^{\prime} d y}{\int_{-L_{0}}^{\infty} w^{2} d y} w=0, \quad y>-L_{0} .
\end{gathered}
$$

Using

$$
\begin{gathered}
\int_{-L_{0}}^{\infty} w^{2} \mathcal{L}^{-1} w d y=\frac{5}{6} \int_{-L_{0}}^{\infty} w^{3} d y+\frac{z}{1-z^{2}} \int_{-L_{0}}^{\infty} w^{2} w^{\prime} d y \\
=3+\frac{45}{8} \int_{0}^{z}\left(1-t^{2}\right)^{2} d t+\frac{z}{1-z^{2}}\left(-\frac{1}{3} w^{3}\left(-L_{0}\right)\right) \\
=3+\frac{45}{8}\left(z-\frac{2}{3} z^{3}+\frac{z^{5}}{5}\right)-\frac{9}{8} z\left(1-z^{2}\right)^{2},
\end{gathered}
$$

this implies

$$
\alpha-2 \alpha \frac{3+\frac{45}{8}\left(z-\frac{2}{3} z^{3}+\frac{z^{5}}{5}\right)-\frac{9}{8} z\left(1-z^{2}\right)^{2}}{3+\frac{3}{2} z\left(3-z^{2}\right)}+\beta \frac{\frac{9}{4}\left(1-z^{2}\right)^{3}}{3+\frac{3}{2} z\left(3-z^{2}\right)}=0
$$

and finally

$$
\alpha=\frac{3\left(1-z^{2}\right)^{3}}{4-2 z\left(3-z^{2}\right)+15\left(z-\frac{2}{3} z^{3}+\frac{z^{5}}{5}\right)-3 z\left(1-z^{2}\right)^{2}} \beta .
$$

Substituting (5.20) into the boundary condition in (5.19), we derive

$\phi_{1}\left(-L_{0}\right)=\alpha\left(\mathcal{L}^{-1} w\right)^{\prime}\left(-L_{0}\right)+\beta w^{\prime \prime}\left(-L_{0}\right)-c\left(\alpha\left(\mathcal{L}^{-1} w\right)\left(-L_{0}\right)+\beta w^{\prime}\left(-L_{0}\right)\right)+\frac{c}{a_{2}} \frac{\int_{\mathbb{R}} w^{2} \phi_{2}}{\int_{\mathbb{R}} w^{2}}=0$.

Elementary computations give

$$
-\frac{3}{4} \beta\left(1-z^{2}\right)^{2}+\frac{c}{a_{2}} \frac{\int_{\mathbb{R}} w^{2} \phi_{2} d y}{\int w^{2} d y}=0
$$

which implies

$$
\beta=\frac{4}{3} \frac{1}{\left(1-z^{2}\right)^{2}} \frac{c}{a_{2}} \frac{\int w^{2} \phi_{2} d y}{\int w^{2} d y} .
$$


Substituting (5.23) into (5.22), we get

$$
\alpha=\frac{4\left(1-z^{2}\right)}{4-2 z\left(3-z^{2}\right)+15\left(z-\frac{2}{3} z^{3}+\frac{z^{5}}{5}\right)-3 z\left(1-z^{2}\right)^{2}} \frac{c}{a_{2}} \frac{\int w^{2} \phi_{2} d y}{\int w^{2} d y}
$$

We now compute the term $2 a_{2} \phi_{1}(0)$ needed in the second equation of (5.19). We decompose

$$
2 a_{2} \phi_{1}(0)=\gamma \frac{\int w \phi_{2} d y}{\int w^{2} d y} .
$$

Then (5.19) has only the trivial kernel if

$$
\gamma \neq 1
$$

(This follows after multiplying NLEP by $w$, integrating, and using a standard result on a local eigenvalue problem. The details of the argument are shown in Section 3 of [33] and for brevity we skip it here.) We compute, using (5.20),

$$
\begin{gathered}
\gamma=2 a_{2}\left(\alpha\left(\mathcal{L}^{-1} w\right)\left(-L_{0}\right)+\beta w^{\prime}\left(-L_{0}\right)\right)\left(\frac{\int w^{2} \phi_{2} d y}{\int w^{2} d y}\right)^{-1} \\
=2 a_{2}\left(\frac{3}{2} \alpha+\frac{3}{2} z\left(1-z^{2}\right) \beta\right) \\
=2 z\left(\frac{6\left(1-z^{2}\right)}{4-2 z\left(3-z^{2}\right)+15\left(z-\frac{2}{3} z^{3}+\frac{z^{5}}{5}\right)-3 z\left(1-z^{2}\right)^{2}}+\frac{2 z}{1-z^{2}}\right) .
\end{gathered}
$$

Now the condition $\gamma \neq 1$ is equivalent to

$$
6 z(z-1)^{2} \neq\left(5 z^{2}-1\right)(z-2) .
$$

This is the same condition as for the operator $\mathcal{L}$ and the monotonicity of the solution graph (compare (5.17)).

\section{Discussion}

Let us finally discuss the biological implications of our results. The patterns observed in our model combine different length scales for different components and are (linearly) stable. This shows that within our model efficient cooperation over different length-scales is possible and the consumer chain can be established in a stable and reliable manner. It confirms that the flow of resources, e.g. raw materials from worldwide mining activities, refinement on a worldwide or national level, production of end-product on a worldwide or national level, finally end-design and sales on a local level can be combined efficiently and reliably.

They will also be important in a biological context to understand spatiotemporal structures in genetic signaling pathways which combine different lengthscales, e.g. at intercellular, intracellular and nucleus level. Morphogens are able to spread information at the various levels by diffusion processes, where smaller diffusivity will lead to smaller lengthscales. Our problem is a simple prototype of such a process in the case of merely two activators. 


\section{Appendix A - Existence II: Rigorous proof}

\section{Completion of the Proof of Theorem 3.1:}

Now we consider the approximate cluster solution which has been introduced in (4.1) and is given by

$$
\begin{array}{r}
\tilde{u}_{1, \epsilon}(x)=\xi_{1}^{\epsilon}\left[w\left(\frac{|x|}{\epsilon_{1}}-L_{0}-r_{\epsilon}\right)\left(1-\chi\left(\frac{|x|}{\epsilon_{1} r_{\epsilon}}\right)\right) \chi(|x|)\right. \\
\left.+\left(\tilde{u}_{1, \epsilon}(0)+\frac{\epsilon_{2}}{\epsilon_{1}} u_{1 a, \epsilon}\left(\frac{|x|}{\epsilon_{2}}\right)\right) \chi\left(\frac{|x|}{\epsilon_{1} r_{\epsilon}}\right)\right] \\
\left.=\tilde{u}_{1 \text { out }, \epsilon} \chi(|x|)+\left(\tilde{u}_{1, \epsilon}(0)+\frac{\epsilon_{2}}{\epsilon_{1}} u_{1 a, \epsilon}\left(\frac{|x|}{\epsilon_{2}}\right)\right) \chi\left(\frac{|x|}{\epsilon_{1} r_{\epsilon}}\right)\right], \\
\tilde{u}_{2, \epsilon}(x)=\xi_{2}^{\epsilon} w\left(\frac{|x|}{\epsilon_{2}}\right) \chi\left(\frac{|x|}{\epsilon_{1} r_{\epsilon}}\right),
\end{array}
$$

where the amplitudes $\xi_{i}, i=1,2$, satisfy (4.3) and (4.2), respectively, $r_{\epsilon}$ is given in (3.4), $\chi$ has been introduced in (3.3), and $u_{1 a, \epsilon}$ is defined in (4.8).

We remark that we use cutoff functions with two different scalings: $\chi(|x|)$ serves to produce an approximate solution which vanishes at the boundary exactly, whereas the role of $\chi\left(\frac{|x|}{\epsilon_{1} r_{\epsilon}}\right)$ is to separate the two small scales. In the following we will sometimes drop the argument of the $\chi$ functions, and, to distinguish the two types we will use the shorthands $\chi_{0}=\chi(|x|)$ and $\chi_{1}=\chi\left(\frac{|x|}{\epsilon_{1} r_{\epsilon}}\right)$. Note that the perturbation caused by $\chi_{0}$ is exponentially small (of order $e^{-C / \epsilon}$ for any $0<C<1$ as $\left.\epsilon \rightarrow 0\right)$ in $\left.L_{(}^{2} \Omega_{\epsilon_{1}}\right)$.

We first compute the error of the approximate cluster solution in system (3.1).

We compute the first component $\tilde{S}_{\epsilon}$ by $\tilde{S}_{\epsilon}=T\left[\tilde{u}_{1, \epsilon}\right]$. and so the first equation of (3.1) is solved exactly.

The second equation of $(3.1)$ at $\left(\tilde{S}_{\epsilon}, \tilde{u}_{1, \epsilon}, \tilde{u}_{2, \epsilon}\right)$ is calculated as follows:

$$
\begin{gathered}
\left(\tilde{u}_{1, \epsilon}\right)^{\prime \prime}-\tilde{u}_{1, \epsilon}+\tilde{S}_{\epsilon} \tilde{u}_{1, \epsilon}^{2}-a_{2} \frac{\epsilon_{1}}{\epsilon_{2}} \tilde{u}_{1, \epsilon} \tilde{u}_{2, \epsilon}^{2} \\
=\left(1-\chi_{1}\right)\left[\tilde{u}_{1, \epsilon}^{\prime \prime}-\tilde{u}_{1, \epsilon}+\tilde{S}_{\epsilon}(0) \tilde{u}_{1, \epsilon}^{2}-a_{2} \frac{\epsilon_{1}}{\epsilon_{2}} \tilde{u}_{1, \epsilon} \tilde{u}_{2, \epsilon}^{2}\right] \\
+\left(1-\chi_{1}\right)\left[\tilde{S}_{\epsilon}-\tilde{S}_{\epsilon}(0)\right] \tilde{u}_{1, \epsilon}^{2} \\
+\chi_{1}\left[\frac{\epsilon_{1}^{2}}{\epsilon_{2}^{2}} \Delta_{y_{2}}\left(\frac{\epsilon_{2}}{\epsilon_{1}} u_{1 a, \epsilon}\right)-\left(\tilde{u}_{1, \epsilon}(0)+\frac{\epsilon_{2}}{\epsilon_{1}} u_{1 a, \epsilon}\right)\right. \\
\left.+\tilde{S}_{\epsilon}\left(\tilde{u}_{1, \epsilon}(0)+\frac{\epsilon_{2}}{\epsilon_{1}} u_{1 a, \epsilon}\right)^{2}-a_{2}\left(\tilde{u}_{1, \epsilon}(0)+\frac{\epsilon_{2}}{\epsilon_{1}} u_{1 a, \epsilon}\right) \tilde{u}_{2, \epsilon}^{2}\right] \\
+2 \chi_{1}^{\prime}\left(u_{1 a, \epsilon, y 1}-\tilde{u}_{1, \epsilon}^{\prime}\right)+\chi_{1}^{\prime \prime}\left(\tilde{u}_{1, \epsilon}(0)+\frac{\epsilon_{2}}{\epsilon_{1}} u_{1 a, \epsilon}-\tilde{u}_{1, \epsilon}\right) \\
=: E_{1}+E_{2}+E_{3}+E_{4} .
\end{gathered}
$$


Here' denotes derivative w.r.t. the variable of the corresponding function, i.e. it means derivative w.r.t. $x$ for $S_{\epsilon}, y_{1}$ for $u_{1}$ and $y_{2}$ for $u_{2}$. We divide $E_{1}=E_{1 a}+E_{1 b}$ into two parts and estimate

$$
\begin{gathered}
E_{1 a}=\left(1-\chi_{1}\right)\left[\tilde{u}_{1, \epsilon}^{\prime \prime}-\tilde{u}_{1, \epsilon}+\tilde{S}_{\epsilon}(0) \tilde{u}_{1, \epsilon}^{2}\right] \\
=O(\epsilon) \quad \text { in } L^{2}\left(\Omega_{\epsilon_{1}}\right) .
\end{gathered}
$$

Here we have used that $\tilde{u}_{1, \epsilon}\left(y_{1}\right)=(1+O(\epsilon)) \frac{1}{\tilde{S}_{\epsilon}(0)} w\left(\left|y_{1}\right|-L_{0}-r_{\epsilon}\right)$ for $\left|y_{1}\right|>0.5 r_{\epsilon}$, and (3.5). The second part of $E_{1}$ is estimated by

$$
E_{1 b}=\left|\left(1-\chi_{1}\right) a_{2} \frac{\epsilon_{1}}{\epsilon_{2}} \tilde{u}_{1, \epsilon} \tilde{u}_{2, \epsilon}^{2}\right| \leq C \frac{\epsilon_{1}}{\epsilon_{2}} w^{2}\left(0.5 \frac{\epsilon_{1}}{\epsilon_{2}} r_{\epsilon}\right)=O\left(\left(\frac{\epsilon_{2}}{\epsilon_{1}}\right)^{9}\right) .
$$

Note for later use that the definition of $r_{\epsilon}$ (see (3.4)) implies

$$
\int_{0}^{\infty} w^{2}\left(y_{2}\right) d y_{2}-\int_{0}^{r_{\epsilon} \epsilon_{1} / \epsilon_{2}} w^{2}\left(y_{2}\right) d y_{2} \sim \int_{r_{\epsilon} \epsilon_{1} / \epsilon_{2}}^{\infty} e^{-2 y_{2}} d y_{2} \sim e^{-2 r_{\epsilon} \epsilon_{1} / \epsilon_{2}} \sim\left(\frac{\epsilon_{2}}{\epsilon_{1}}\right)^{20} .
$$

Computing $\tilde{S}_{\epsilon}(x)$ using the Green's function $G_{D}$ introduced in (9.1), we derive the following estimate:

$$
\begin{gathered}
E_{2}=\left[\tilde{S}_{\epsilon}\left(\epsilon_{1} y_{1}\right)-\tilde{S}_{\epsilon}(0)\right] \tilde{u}_{1, \epsilon}^{2} \\
=\tilde{u}_{1, \epsilon}^{2} a_{1} \int_{-1 / \epsilon_{1}}^{1 / \epsilon_{1}}\left[G_{D}\left(\epsilon_{1} y_{1}, \epsilon_{1} z\right)-G_{D}\left(0, \epsilon_{1} z\right)\right] \tilde{S}_{\epsilon}(z) \tilde{u}_{1, \epsilon}^{2}(z) d z\left(1+O\left(\epsilon_{1}\right)\right) \\
=a_{1} \frac{\tilde{u}_{1, \epsilon}^{2}}{\tilde{S}_{\epsilon}(0)} \epsilon_{1} \int_{\mathbb{R}}\left(\frac{1}{2 D}\left|y_{1}-z_{1}\right|-\frac{1}{2 D}\left|z_{1}\right|\right) w^{2}\left(|z|-L_{0}\right) d z\left(1+O\left(r_{\epsilon}+\epsilon_{1}\left|y_{1}\right|\right)\right) \\
+a_{1} \frac{\tilde{u}_{1, \epsilon}^{2}}{\tilde{S}_{\epsilon}(0)} \epsilon_{1}^{2} y_{1}^{2} \nabla^{2} H_{D}(0,0)\left(6+2 \rho\left(L_{0}\right)\right)\left(1+O\left(r_{\epsilon}+\epsilon_{1}\left|y_{1}\right|\right)\right) \\
=O\left(\epsilon_{1}\left|y_{1}\right| \tilde{u}_{1, \epsilon}^{2}\right)=O(\epsilon) \quad \text { in } L^{2}\left(\Omega_{\epsilon_{1}}\right) .
\end{gathered}
$$

Note that $\nabla H_{D}(0,0)=0$ by symmetry. (See the computation of $H_{D}$ in the appendix).

On the small scale, we estimate

$$
\begin{gathered}
E_{3}=\chi_{1}\left[\frac{\epsilon_{1}^{2}}{\epsilon_{2}^{2}} \Delta_{y_{2}} \frac{\epsilon_{2}}{\epsilon_{1}} u_{1 a, \epsilon}-\left(\tilde{u}_{1, \epsilon}(0)+\frac{\epsilon_{2}}{\epsilon_{1}} u_{1 a, \epsilon}\right)\right. \\
\left.+\tilde{S}_{\epsilon}\left(\tilde{u}_{1, \epsilon}(0)+\frac{\epsilon_{2}}{\epsilon_{1}} u_{1 a, \epsilon}\right)^{2}-a_{2}\left(\frac{\epsilon_{1}}{\epsilon_{2}} \tilde{u}_{1, \epsilon}(0)+u_{1 a, \epsilon}\right) \tilde{u}_{2, \epsilon}^{2}\right] \\
=\chi_{1} \frac{\epsilon_{1}}{\epsilon_{2}}\left[\Delta_{y_{2}} u_{1 a, \epsilon}-a_{2} \tilde{u}_{1, \epsilon}(0) \tilde{u}_{2, \epsilon}^{2}\right] \\
-\tilde{u}_{1, \epsilon}\left(r_{\epsilon}\right)+\tilde{S}_{\epsilon} \tilde{u}_{1, \epsilon}^{2}\left(r_{\epsilon}\right)+O(\epsilon) \\
=O(\epsilon) \quad \text { in } L^{2}\left(\Omega_{\epsilon_{2}}\right)
\end{gathered}
$$

by the definition of $u_{1 a, \epsilon}$ given in (4.8) and due to the relation between $\xi_{1}=\tilde{u}_{1, \epsilon}(0)$ and $\tilde{S}_{\epsilon}\left(r_{\epsilon}\right)$ given in (4.3).

Finally, we estimate the error on the overlapping region between inner and outer scale for which $\chi_{1}^{\prime} \neq 0$ and is contained in $0.5 r_{\epsilon}<y_{1}<r_{\epsilon}$. 
The exact matching conditions at $x=r_{\epsilon}$ are

$$
\tilde{u}_{1, \epsilon}(0)+\frac{\epsilon_{2}}{\epsilon_{1}} u_{1 a, \epsilon}\left(\frac{\epsilon_{1}}{\epsilon_{2}} r_{\epsilon}\right)-\tilde{u}_{1 o u t, \epsilon}\left(r_{\epsilon}\right)=0
$$

and

$$
u_{1 a, \epsilon, y_{2}}\left(\frac{\epsilon_{1}}{\epsilon_{2}} r_{\epsilon}\right)-\tilde{u}_{1 o u t, \epsilon}^{\prime}\left(r_{\epsilon}\right)=0 .
$$

Note that the system (7.1), (7.2) in leading order is given by

$$
\begin{gathered}
c_{1, \epsilon} \frac{\epsilon_{1}}{\epsilon_{2}} r_{\epsilon}+c_{2, \epsilon} e^{-r_{\epsilon} \epsilon_{1} / \epsilon_{2}}+c_{4, \epsilon}=c_{3, \epsilon} w\left(-L_{0}\right) \\
c_{1, \epsilon}-c_{2, \epsilon} e^{-r_{\epsilon} \epsilon_{1} / \epsilon_{2}}=c_{3, \epsilon} w_{y_{1}}\left(-L_{0}\right),
\end{gathered}
$$

where $c_{1, \epsilon}, c_{2, \epsilon}, c_{3, \epsilon}$ are given constants which have limits as $\epsilon \rightarrow 0, r_{\epsilon}$ is given by (3.4), and the unknowns are $c_{4, \epsilon}$ and $L_{0}$. The system (7.1), (7.2) can be solved exactly and it has a unique solution.

This implies

$$
\tilde{u}_{1, \epsilon}(0)+\frac{\epsilon_{2}}{\epsilon_{1}} u_{1 a, \epsilon}\left(\frac{\epsilon_{1}}{\epsilon_{2}} y_{1}\right)-\tilde{u}_{1 o u t, \epsilon}\left(y_{1}\right)=O\left(\left(y_{1}-r_{\epsilon}\right)^{2}\right)
$$

and

$$
u_{1 a, \epsilon, y_{2}}\left(\frac{\epsilon_{1}}{\epsilon_{2}} y_{1}\right)-\tilde{u}_{1 o u t, \epsilon}^{\prime}=O\left(\left|y_{1}-r_{\epsilon}\right|\right)
$$

Combining this with

$$
\left|\chi_{1}\right| \leq 1, \quad\left|\chi_{1}^{\prime}\right| \leq \frac{C}{r_{\epsilon}}, \quad\left|\chi_{1}^{\prime \prime}\right| \leq \frac{C}{r_{\epsilon}^{2}}
$$

we derive

$$
\left|\chi_{1}^{\prime} \tilde{u}_{1, \epsilon}^{\prime}\right| \leq C, \quad\left|\chi_{1}^{\prime \prime} \tilde{u}_{1, \epsilon}\right| \leq C .
$$

With this knowledge in hand, we can now easily estimate

$$
\begin{gathered}
E_{4}=2 \chi_{1}^{\prime}\left(u_{1 a, \epsilon, y_{1}}-\tilde{u}_{1, \epsilon}^{\prime}\right)+\chi_{1}^{\prime \prime}\left(\tilde{u}_{1, \epsilon}(0)+\frac{\epsilon_{2}}{\epsilon_{1}} u_{1 a, \epsilon}-\tilde{u}_{1, \epsilon}\right) \\
=O(\epsilon) \quad \text { in } L^{2}\left(\Omega_{\epsilon_{2}}\right) .
\end{gathered}
$$

The third equation in (3.1) becomes

$$
\begin{gathered}
\Delta_{y_{2}} \tilde{u}_{2, \epsilon}-\tilde{u}_{2, \epsilon}+\tilde{u}_{1, \epsilon} \tilde{u}_{2 \epsilon}^{2} \\
=\Delta_{y_{2}} \tilde{u}_{2, \epsilon}-\tilde{u}_{2, \epsilon}+\tilde{u}_{1, \epsilon}(0) \tilde{u}_{2 \epsilon}^{2} \\
+\left[\tilde{u}_{1, \epsilon}-\tilde{u}_{1, \epsilon}(0)\right] \tilde{u}_{2 \epsilon}^{2} \\
=O(\epsilon)+O\left(\frac{\epsilon_{2}}{\epsilon_{1}}\left|y_{2}\right| \tilde{u}_{2 \epsilon}^{2}\right) \\
=O(\epsilon) \quad \text { in } L^{2}\left(\Omega_{\epsilon_{2}}\right)
\end{gathered}
$$

by using Taylor expansion of $\tilde{u}_{1, \epsilon}$ at 0 .

We introduce the following vectorial $L^{2}$ norm

$$
\left\|\left|\left(\phi_{1}, \phi_{2}\right)\left\|\left.\right|_{L^{2}} ^{2}=\right\| \chi_{1} \phi_{1}\left\|_{L^{2}\left(\Omega_{\epsilon_{2}}\right)}^{2}+\right\|\left(1-\chi_{1}\right) \phi_{1}\left\|_{L^{2}\left(\Omega_{\epsilon_{1}}\right)}^{2}+\right\| \phi_{2} \|_{L^{2}\left(\Omega_{\epsilon_{2}}\right)}^{2} .\right.\right.
$$


Similarly, for the $H^{2}$ norm we define

$$
\left\|\left|\left(\phi_{1}, \phi_{2}\right)\left\|\left.\right|_{H^{2}} ^{2}=\right\| \chi_{1} \phi_{1}\left\|_{H^{2}\left(\Omega_{\epsilon_{2}}\right)}^{2}+\right\|\left(1-\chi_{1}\right) \phi_{1}\left\|_{H^{2}\left(\Omega_{\epsilon_{1}}\right)}^{2}+\right\| \phi_{2} \|_{H^{2}\left(\Omega_{\epsilon_{2}}\right)}^{2} .\right.\right.
$$

Writing the system (3.1) as $R_{\epsilon}\left(S, u_{1}, u_{2}\right)=0$, we have now shown the estimate

$$
\left\|\mid R_{\epsilon}\left(T\left[\tilde{u}_{1, \epsilon}\right], \tilde{u}_{1, \epsilon}, \tilde{u}_{2, \epsilon}\right)\right\|_{L^{2}}=O(\epsilon) .
$$

(Note that the first equation is solved exactly and the first component does not enter in the definition of the norm.)

Next study the linearized operator $\tilde{\mathcal{L}}_{\epsilon}$ around the approximate solution $\left(\tilde{S}_{\epsilon}, \tilde{u}_{\epsilon, 1}, \tilde{u}_{\epsilon, 2}\right)$. It is defined as follows

$$
\begin{gathered}
\tilde{\mathcal{L}}_{\epsilon}:\left(H_{N}^{2}(\Omega)\right)^{3} \rightarrow\left(L^{2}(\Omega)\right)^{3}, \quad \tilde{\mathcal{L}}_{\epsilon}\left(\begin{array}{c}
\psi_{\epsilon} \\
\phi_{1, \epsilon} \\
\phi_{2, \epsilon}
\end{array}\right)= \\
=\left(\begin{array}{l}
D \Delta \psi_{\epsilon}-2 \frac{a_{1}}{\epsilon_{1}} \tilde{S}_{\epsilon} \tilde{u}_{1, \epsilon} \phi_{1, \epsilon}-\frac{a_{1}}{\epsilon_{1}} \psi_{\epsilon} \tilde{u}_{1, \epsilon}^{2} \\
\epsilon_{1}^{2} \Delta \phi_{1, \epsilon}-\phi_{1, \epsilon}+2 \tilde{S}_{\epsilon} \tilde{u}_{1, \epsilon} \phi_{1, \epsilon}+\psi_{\epsilon} \tilde{u}_{1, \epsilon}^{2}-a_{2} \frac{\epsilon_{1}}{\epsilon_{2}} \phi_{1, \epsilon} \tilde{u}_{2, \epsilon}^{2}-2 a_{2} \frac{\epsilon_{1}}{\epsilon_{2}} \tilde{u}_{1, \epsilon} \tilde{u}_{2, \epsilon} \phi_{2, \epsilon} \\
\epsilon_{2}^{2} \Delta \phi_{2, \epsilon}-\phi_{2, \epsilon}+\phi_{1, \epsilon} \tilde{u}_{2, \epsilon}^{2}+2 \tilde{u}_{1, \epsilon} \tilde{u}_{2, \epsilon} \phi_{2, \epsilon}
\end{array}\right) .
\end{gathered}
$$

When discussing the kernel of $\tilde{\mathcal{L}}_{\epsilon}$ we first determine $\psi=T^{\prime}\left[\phi_{1}\right]$ using the Green's function $G_{D, \tau \lambda_{\epsilon}}$ defined in (9.7). Therefore we study instead the following operator $\overline{\mathcal{L}}_{\epsilon}$ which is applied to the second and third components. Further, to have uniform invertibility we have to introduce suitable approximate kernel and co-kernel given by

$$
\begin{gathered}
\mathcal{K}_{\epsilon}=\operatorname{span}\left\{\tilde{u}_{2, \epsilon}^{\prime}\right\} \subset H_{N}^{2}\left(\Omega_{\epsilon_{2}}\right), \\
\mathcal{C}_{\epsilon}=\operatorname{span}\left\{\tilde{u}_{2, \epsilon}^{\prime}\right\} \subset L^{2}\left(\Omega_{\epsilon_{2}}\right) .
\end{gathered}
$$

Then the linear operator $\overline{\mathcal{L}}_{\epsilon}$ is defined by

$$
\overline{\mathcal{L}}_{\epsilon}: H_{N}^{2}\left(\Omega_{\epsilon_{1}}\right) \oplus \mathcal{K}_{\epsilon}^{\perp} \rightarrow L^{2}\left(\Omega_{\epsilon_{1}}\right) \oplus \mathcal{C}_{\epsilon}^{\perp},
$$

$\overline{\mathcal{L}}_{\epsilon}\left(\begin{array}{c}\phi_{1, \epsilon} \\ \phi_{2, \epsilon}\end{array}\right)=\left(\begin{array}{l}\Delta_{y_{1}} \phi_{1, \epsilon}-\phi_{1, \epsilon}+2 \tilde{S}_{\epsilon} \tilde{u}_{1, \epsilon} \phi_{1, \epsilon}+T^{\prime}\left[\phi_{1, \epsilon}\right] \tilde{u}_{1, \epsilon}^{2}-a_{2} \frac{\epsilon_{1}}{\epsilon_{2}} \phi_{1, \epsilon} \tilde{u}_{2, \epsilon}^{2}-2 a_{2} \frac{\epsilon_{1}}{\epsilon_{2}} \tilde{u}_{1, \epsilon} \tilde{u}_{2, \epsilon} \phi_{2, \epsilon} \\ \Delta_{y_{2}} \phi_{2, \epsilon}-\phi_{2, \epsilon}+\phi_{1, \epsilon} \tilde{u}_{2, \epsilon}^{2}+2 \tilde{u}_{1, \epsilon} \tilde{u}_{2, \epsilon} \phi_{2, \epsilon}\end{array}\right)$.

where $\perp$ means perpendicular in $L^{2}$ sense. Now we show that this operator is uniformly invertible for $\epsilon$ small enough. In fact, we have the following result:

Proposition 7.1. There exist positive constants $\bar{\epsilon}, \lambda$ such that for all $\epsilon \in(0, \bar{\epsilon})$,

$$
\left\|\left|\overline{\mathcal{L}}_{\epsilon}\left(\phi_{1}, \phi_{2}\right)\left\|\left.\right|_{L_{2}} \geq \lambda\right\|\left\|\left(\phi_{1}, \phi_{2}\right)\right\|\right|_{H^{2}} \quad \text { for all }\left(\phi_{1}, \phi_{2}\right) \in H_{N}^{2}\left(\Omega_{\epsilon_{1}}\right) \oplus \mathcal{K}_{\epsilon}^{\perp} .\right.
$$

Further, the linear operator $\overline{\mathcal{L}}_{\epsilon}$ is surjective with the norms introduced in (7.5), (7.6). 
Proof of Proposition 7.1. We give an indirect proof. Suppose (7.10) is false. Using the notation $\Phi_{\epsilon}=\left(\begin{array}{c}\phi_{1, \epsilon} \\ \phi_{2, \epsilon}\end{array}\right)$ and employing the norms denoted $\||\Phi \||$ and introduced in (7.5), (7.6), then there exist sequences $\left\{\epsilon_{k}\right\},\left\{\Phi^{k}\right\}$ with $\epsilon_{k} \rightarrow 0, \Phi^{k}=\Phi_{\epsilon_{k}}, k=1,2, \ldots$ such that

$$
\begin{aligned}
& \left\|\mid \overline{\mathcal{L}}_{\epsilon_{k}} \Phi^{k}\right\|_{L^{2}} \rightarrow 0, \quad \text { as } k \rightarrow \infty, \\
& \left\|\mid \Phi^{k}\right\| \|_{H^{2}}=1, \quad k=1,2, \ldots
\end{aligned}
$$

Using the cutoff function defined in (3.3), we introduce the following functions:

$$
\begin{gathered}
\phi_{1 a, \epsilon}\left(y_{1}\right)=\phi_{1, \epsilon}\left(y_{1}\right), \quad r_{\epsilon} \leq\left|y_{1}\right| \leq \frac{1}{\epsilon_{1}} . \\
\phi_{1 b, \epsilon}\left(y_{2}\right)=\frac{\epsilon_{1}}{\epsilon_{2}}\left(\phi_{1, \epsilon}\left(\frac{\epsilon_{2}}{\epsilon_{1}} y_{2}\right)-\phi_{1, \epsilon}(0)\right), \quad\left|y_{2}\right| \leq \frac{\epsilon_{1}}{\epsilon_{2}} r_{\epsilon} .
\end{gathered}
$$

Because of (7.1), (7.2) we have

$$
\phi_{1 b, \epsilon}\left(\frac{\epsilon_{1}}{\epsilon_{2}} r_{\epsilon}\right)-\phi_{1 a, \epsilon}\left(r_{\epsilon}\right)=0
$$

and

$$
\phi_{1 b, \epsilon, y_{2}}\left(\frac{\epsilon_{1}}{\epsilon_{2}} r_{\epsilon}\right)-\phi_{1 a, \epsilon, y_{2}}\left(r_{\epsilon}\right)=0
$$

At first (after rescaling) the functions $\phi_{1 a, \epsilon}, \phi_{1 b, \epsilon}, \phi_{2, \epsilon}$ are only defined in for $r_{\epsilon} \leq\left|y_{1}\right| \leq \frac{1}{\epsilon_{1}}$, $\left|y_{2}\right| \leq \frac{\epsilon_{1}}{\epsilon_{2}} r_{\epsilon}$ and $\left|y_{2}\right| \leq \frac{1}{\epsilon_{2}}$, respectively. However, by a standard result, $\phi_{1 a, \epsilon}$ can be extended to $\mathbb{R} \backslash\{0\}$ and $\phi_{1 b, \epsilon}, \phi_{2, \epsilon}$ can be extended to $\mathbb{R}$ such that the norms of $\phi_{1 a, \epsilon}$ in $H^{2}(\mathbb{R} \backslash\{0\})$, $\phi_{1 b, \epsilon}$ locally in $H^{2}(\mathbb{R})$ (on any bounded domain) and $\phi_{2, \epsilon}$ in $H^{2}(\mathbb{R})$, respectively, are bounded by a constant independent of $\epsilon$ for all $\epsilon$ small enough. In the following we will study this extension. For simplicity of notation we keep the same notation for the extension. Since for $i=1 b, 2$ each sequence $\left\{\phi_{i}^{k}\right\}:=\left\{\phi_{i, \epsilon_{k}}\right\}(k=1,2, \ldots)$ is bounded in $H_{l o c}^{2}(\mathbb{R})$ it has a weak limit in $H_{\text {loc }}^{2}(\mathbb{R})$, and therefore also a strong limit in $L_{\text {loc }}^{2}(\mathbb{R})$ and $L_{l o c}^{\infty}(\mathbb{R})$. (For $i=1 a$, the same argument holds with $\mathbb{R}$ replaced by $\mathbb{R} \backslash\{0\}$.) Call these limits $\phi_{i}$. Then, taking the limit $\epsilon \rightarrow 0$ in $(7.9)$, we derive that $\Phi=\left(\begin{array}{c}\phi_{1 a} \\ \phi_{1 b} \\ \phi_{2}\end{array}\right)$ satisfies

$$
\int_{\mathbb{R}} \phi_{2} w_{y_{2}} d y_{2}=0
$$

and solves the system

$$
\mathcal{L} \Phi=0,
$$


where the operator $\mathcal{L}$ is defined as follows

$$
\mathcal{L}\left(\begin{array}{c}
\phi_{1 a} \\
\phi_{1 b} \\
\phi_{2}
\end{array}\right)=\left(\begin{array}{c}
\Delta_{y_{1}} \phi_{1 a}-\phi_{1 a}+2 w_{L_{0}} \phi_{1 a}-\frac{2 \int_{0}^{\infty} w_{L_{0}} \phi_{1}}{\int_{0}^{\infty} w_{L_{0}}^{2}} w_{L_{0}}^{2}, \quad y_{1} \neq 0 \\
\phi_{1 b}\left(y_{2}\right)-a_{2} \frac{\phi_{1 b}(0)}{u_{1}^{2}(0)} \int_{0}^{y_{2}} \int_{0}^{t} w^{2} d t d y_{2}-2 a_{2} \int_{0}^{y_{2}} \int_{0}^{t} w \phi_{2} d t d y_{2} \\
\Delta_{y_{2}} \phi_{2}-\phi_{2}+2 w \phi_{2}+\frac{\phi_{1 b}(0)}{\left(u_{1}(0)\right)^{2}} w^{2}, \quad y_{2} \in \mathbb{R}
\end{array}\right)
$$

with $w_{L_{0}}(|y|)=w\left(|y|-L_{0}\right)$.

The matching condition

$$
\phi_{1 b}^{\prime}( \pm \infty)=\phi_{1 a}^{\prime}( \pm 0)
$$

follows from (7.14) and it implies the boundary condition (5.6).

The matching condition

$$
\phi_{1 b}( \pm \infty)=\phi_{1 a}(0)
$$

follows from (7.15) and it determines $\phi_{1 b}(0)$.

By Lemma 5.1, it follows for $L_{0} \neq L_{0}^{m}$ that $\Phi=0$.

By elliptic estimates we get $\left\|\phi_{i, \epsilon_{k}}\right\|_{H^{2}(\mathbb{R})} \rightarrow 0$ as $k \rightarrow \infty$. for $i=1 b, 2$ and $\left\|\phi_{1 a, \epsilon_{k}}\right\|_{H^{2}(\mathbb{R} \backslash\{0\})} \rightarrow$ 0 as $k \rightarrow \infty$.

This contradicts $\left\|\left|\Phi^{k} \|\right|_{H^{2}}=1\right.$. To complete the proof of Proposition 7.1, we need to show that the adjoint operator to $L_{\epsilon}$ (denoted by $L_{\epsilon}^{*}$ ) is injective from $H^{2}$ to $L^{2}$.

The limiting process as $\epsilon \rightarrow 0$ for the adjoint operator $\overline{\mathcal{L}}_{\epsilon}^{*}$ follows exactly along the same lines as the proof for $\overline{\mathcal{L}}_{\epsilon}$ and is therefore omitted. By Lemma 5.2, the limiting adjoint operator $\mathcal{L}^{*}$ has only the trivial kernel.

Finally, we solve the system (3.1), which we write as

$$
R_{\epsilon}\left(\tilde{S}_{\epsilon}+\psi, \tilde{u}_{1, \epsilon}+\phi_{1}, \tilde{u}_{2, \epsilon}+\phi_{2}\right)=R_{\epsilon}\left(U_{\epsilon}+\Phi\right)=0,
$$

using the notation $U_{\epsilon}=\left(\tilde{S}_{\epsilon}, \tilde{u}_{1, \epsilon}, \tilde{u}_{2, \epsilon}\right)$. By Proposition 7.1, for $\epsilon$ small enough we can write (7.19) as follows:

$$
\Phi=-\mathcal{L}_{\epsilon}^{-1} R_{\epsilon}\left(U_{\epsilon}\right)-\mathcal{L}_{\epsilon}^{-1} N_{\epsilon}(\Phi)=: M_{\epsilon}(\Phi)
$$

where

$$
N_{\epsilon}(\Phi)=R_{\epsilon}\left(U_{\epsilon}+\Phi\right)-R_{\epsilon}\left(U_{\epsilon}\right)-R_{\epsilon}^{\prime}\left(U_{\epsilon}\right) \Phi
$$

and the operator $M_{\epsilon}$ defined by (7.20) is a mapping from $H^{2}$ into itself. We are going to show that the operator $M_{\epsilon}$ is a contraction on

$$
B_{\epsilon, \delta} \equiv\left\{\phi \in H^{2}:\|\mid \phi\|_{H^{2}}<\delta\right\}
$$

if $\delta$ and $\epsilon$ are suitably chosen. By (7.7) and Proposition 7.1 we have

$$
\begin{gathered}
\left\|\mid M_{\epsilon}(\Phi)\right\| \|_{H^{2}} \leq \lambda^{-1}\left(\left\|\left|N_{\epsilon}(\Phi)\left\|\left.\right|_{L^{2}}+\right\|\right| R_{\epsilon}\left(U_{\epsilon}\right)\right\|_{L^{2}}\right) \\
\leq \lambda^{-1} C(c(\delta) \delta+\epsilon),
\end{gathered}
$$


where $\lambda>0$ is independent of $\delta>0, \epsilon>0$ and $c(\delta) \rightarrow 0$ as $\delta \rightarrow 0$. Similarly we show

$$
\left.\left\|\left|M_{\epsilon}\left(\Phi_{1}\right)-M_{\epsilon}\left(\Phi_{2}\right)\left\|\left.\right|_{H^{2}} \leq \lambda^{-1} C(c(\delta) \delta)\right\|\right| \Phi_{1}-\Phi_{2}\right\|\right|_{H^{2}},
$$

where $c(\delta) \rightarrow 0$ as $\delta \rightarrow 0$. If we choose $\delta=c_{3} \epsilon$, then, for suitable $c_{3}>0$ and $\epsilon$ small enough, $M_{\epsilon}$ is a contraction on $B_{\epsilon, \delta}$. The existence of a fixed point $\Phi_{\epsilon}$ now follows from the standard contraction mapping principle, and $\Phi_{\epsilon}$ is a solution of (7.20).

We have thus proved

Lemma 7.1. There exists $\bar{\epsilon}>0$ such that for every $\epsilon$ with $0<\epsilon<\bar{\epsilon}$ there is a unique $\Phi_{\epsilon} \in H^{2}$ satisfying $R_{\epsilon}\left(U_{\epsilon}+\Phi_{\epsilon}\right)=0$. Furthermore, we have the estimate

$$
\left\|\mid \Phi_{\epsilon}\right\|_{H^{2}} \leq C \epsilon .
$$

This completes the proof of Theorem 3.1.

In this section we have constructed an exact cluster solution of the form $U_{\epsilon}+\Phi_{\epsilon}=$ $\left(S_{\epsilon}, u_{\epsilon, 1}, u_{\epsilon, 2}\right)$. In the next section we are going to study its stability.

\section{Appendix B - Stability II: Computation of the small eigenvalues}

\section{Completion of the Proof of Theorem 3.2:}

We compute the small eigenvalues of the eigenvalue problem (5.3), i.e. we determine the eigenvalues assuming that $\lambda_{\epsilon} \rightarrow 0$ as $\epsilon \rightarrow 0$. We will prove that they satisfy $\lambda_{\epsilon}=O\left(\epsilon_{2}^{2}\right)$. Let us define

$$
\tilde{u}_{i, \epsilon}(x)=\chi(|x|) u_{i, \epsilon}(x) .
$$

Then it follows easily that $\tilde{u}_{i, \epsilon}$ in $H_{N}^{2}\left(\Omega_{\epsilon_{i}}\right)$ and

$$
u_{i, \epsilon}(x)=\tilde{u}_{i, \epsilon}(x)+\text { e.s.t. } \quad \text { in } H^{2}\left(\Omega_{\epsilon_{i}}\right) .
$$

Taking the derivative of the system (3.1) w.r.t. $y_{2}$, we compute

$$
\left\{\begin{array}{l}
\epsilon_{1}^{2} \Delta \frac{\epsilon_{2}}{\epsilon_{1}} \tilde{u}_{1, \epsilon}^{\prime}-\frac{\epsilon_{2}}{\epsilon_{1}} \tilde{u}_{1, \epsilon}^{\prime}+2 S_{\epsilon} u_{1, \epsilon} \frac{\epsilon_{2}}{\epsilon_{1}} \tilde{u}_{1, \epsilon}^{\prime}+\epsilon_{2} S_{\epsilon}^{\prime} u_{1, \epsilon}^{2}-a_{2} \frac{\epsilon_{1}}{\epsilon_{2}} \frac{\epsilon_{2}}{\epsilon_{1}} \tilde{u}_{1, \epsilon}^{\prime} u_{2, \epsilon}^{2}-2 a_{2} \frac{\epsilon_{1}}{\epsilon_{2}} u_{1, \epsilon} u_{2, \epsilon} \tilde{u}_{2, \epsilon}^{\prime}=\text { e.s.t. } \\
\epsilon_{2}^{2} \Delta \tilde{u}_{2, \epsilon}^{\prime}-\tilde{u}_{2, \epsilon}^{\prime}+\frac{\epsilon_{2}}{\epsilon_{1}} \tilde{u}_{1, \epsilon}^{\prime} u_{2, \epsilon}^{2}+2 u_{1, \epsilon} u_{2, \epsilon} \tilde{u}_{2, \epsilon}^{\prime}=\text { e.s.t. }
\end{array}\right.
$$

Let us now decompose the eigenfunction $\left(\psi_{\epsilon}, \phi_{1, \epsilon}, \phi_{2, \epsilon}\right)$ as follows:

$$
\phi_{1, \epsilon}=a^{\epsilon} \frac{\epsilon_{2}}{\epsilon_{1}} \tilde{u}_{1, \epsilon}^{\prime}+\bar{\phi}_{1, \epsilon}
$$

where $a^{\epsilon}$ is a complex number to be determined and

$$
\begin{aligned}
& \bar{\phi}_{1, \epsilon} \in H_{N}^{2}\left(\Omega_{\epsilon_{1}}\right) \text {; } \\
& \phi_{2, \epsilon}=a^{\epsilon} \tilde{u}_{2, \epsilon}^{\prime}+\bar{\phi}_{2, \epsilon} \sim \frac{a^{\epsilon}}{u_{1, \epsilon}(0)} w_{L}^{\prime} \chi_{0}+\bar{\phi}_{2, \epsilon},
\end{aligned}
$$

where

$$
\bar{\phi}_{2, \epsilon}=\bar{\phi}_{2 a, \epsilon}+\phi_{2, \epsilon}^{\perp}
$$




$$
\bar{\phi}_{2 a, \epsilon} \in H_{N}^{2}\left(\Omega_{\epsilon_{2}}\right), \quad \phi_{2, \epsilon}^{\perp} \perp \mathcal{K}_{\epsilon}=\operatorname{span}\left\{\tilde{u}_{2, \epsilon}^{\prime}\right\} \subset H_{N}^{2}\left(\Omega_{\epsilon_{2}}\right) .
$$

We decompose the eigenfunction $\psi_{\epsilon}$ as follows:

$$
\psi_{\epsilon}=a^{\epsilon} \psi_{1, \epsilon}+\bar{\psi}_{\epsilon}
$$

where $\psi_{1, \epsilon}$ satisfies

$$
\left\{\begin{array}{c}
D \Delta \psi_{1, \epsilon}-\frac{a_{1}}{\epsilon_{1}} \psi_{1, \epsilon} u_{1, \epsilon}^{2}-2 \frac{a_{1}}{\epsilon_{1}} S_{\epsilon} u_{1, \epsilon} \frac{\epsilon_{2}}{\epsilon_{1}} \tilde{u}_{1, \epsilon}^{\prime}=\tau \lambda_{\epsilon} \psi_{1, \epsilon}, \\
\psi_{1, \epsilon}^{\prime}( \pm 1)=0
\end{array}\right.
$$

and $\bar{\psi}_{\epsilon}$ is given by

$$
\left\{\begin{array}{c}
D \Delta \bar{\psi}_{\epsilon}-\frac{a_{1}}{\epsilon_{1}} \bar{\psi}_{\epsilon} u_{1, \epsilon}^{2}-2 \frac{a_{1}}{\epsilon_{1}} S_{\epsilon} u_{1, \epsilon} \bar{\phi}_{1, \epsilon}=\tau \lambda_{\epsilon} \bar{\psi}_{\epsilon}, \\
\left(\bar{\psi}_{\epsilon}\right)^{\prime}( \pm 1)=0 .
\end{array}\right.
$$

Note that $\psi_{\epsilon}$ can be uniquely expressed in terms of $\phi_{1, \epsilon}$ using the Green's function $G_{D, \tau \lambda_{\epsilon}}$ given in (9.7):

$$
\psi_{\epsilon}=a^{\epsilon} \psi_{1, \epsilon}+\bar{\psi}_{\epsilon}=a^{\epsilon} T_{\tau \lambda_{\epsilon}}^{\prime}\left[\frac{\epsilon_{2}}{\epsilon_{1}} \tilde{u}_{1, \epsilon}^{\prime}\right]+T_{\tau \lambda_{\epsilon}}^{\prime}\left[\bar{\phi}_{1, \epsilon}\right] .
$$

The derivative $S_{\epsilon}^{\prime}$ satisfies

$$
D\left(S_{\epsilon}^{\prime}\right)^{\prime \prime}-\frac{a_{1}}{\epsilon_{1}} S_{\epsilon}^{\prime} u_{1, \epsilon}^{2}-2 \frac{a_{1}}{\epsilon_{1}} S_{\epsilon} u_{1, \epsilon} \frac{1}{\epsilon_{1}} \tilde{u}_{1, \epsilon}^{\prime}=\text { e.s.t., } \quad S_{\epsilon}^{\prime} \in H^{2}(-1,1), S_{\epsilon}^{\prime}(-1)=S_{\epsilon}^{\prime}(1)=0 .
$$

Using the following Green's function for Dirichlet boundary conditions

$$
D G_{p}^{\prime \prime}=\delta_{z}, x \in(-1,1), \quad G_{p}(-1)=G_{p}(1)=0
$$

which is given by

$$
G_{p}(x, z)=\frac{1}{2 D}|x-z|+\frac{1}{2 D}(x z-1)
$$

we compute $S_{\epsilon}^{\prime}$ near zero. We get

$$
\begin{gathered}
S_{\epsilon}^{\prime}\left(\epsilon_{1} y_{1}\right)-S_{\epsilon}^{\prime}(0) \\
=a_{1} \int_{-1 / \epsilon_{1}}^{1 / \epsilon_{1}}\left[G_{p}\left(\epsilon_{1} y_{1}, \epsilon_{1} z\right)-G_{p}\left(0, \epsilon_{1} z\right)\right]\left(S_{\epsilon}^{\prime} u_{1, \epsilon}^{2}+2 S_{\epsilon} u_{1, \epsilon} \frac{1}{\epsilon_{1}} \tilde{u}_{1, \epsilon}^{\prime}\right) d z_{1}\left(1+O\left(\epsilon_{1}\right)\right) \\
=a_{1} \int_{-1 / \epsilon_{1}}^{1 / \epsilon_{1}}\left[\frac{1}{2 D} \epsilon_{1}\left(\left|y_{1}-z_{1}\right|-\left|z_{1}\right|\right)+\frac{1}{2 D}\left(\epsilon_{1}^{2} y_{1} z_{1}\right)\right]\left(S_{\epsilon}^{\prime} u_{1, \epsilon}^{2}+2 S_{\epsilon} u_{1, \epsilon} \frac{1}{\epsilon_{1}} \tilde{u}_{1, \epsilon}^{\prime}\right) d z_{1}\left(1+O\left(\epsilon_{1}\right)\right) \\
=\frac{a_{1}}{D}\left[\int_{-1 / \epsilon_{1}}^{1 / \epsilon_{1}}\left(\left|y_{1}-z_{1}\right|-\left|z_{1}\right|\right) S_{\epsilon} u_{1, \epsilon} \frac{1}{\epsilon_{1}} \tilde{u}_{1, \epsilon}^{\prime} d z_{1}+\frac{\epsilon_{1}}{S_{\epsilon}(0)} y_{1} \int_{-L_{0}-r_{\epsilon}}^{\infty} z_{1} 2 w w^{\prime} d z_{1}\right](1+O(\epsilon)) \\
=\frac{a_{1}}{D}\left[\int_{-1 / \epsilon_{1}}^{1 / \epsilon_{1}}\left(\left|y_{1}-z_{1}\right|-\left|z_{1}\right|\right) S_{\epsilon} u_{1, \epsilon} \frac{1}{\epsilon} \tilde{u}_{1, \epsilon}^{\prime} d z_{1}-\frac{\epsilon_{1}}{S_{\epsilon}(0)}\left(1+O\left(r_{\epsilon}\right)\right) y_{1}\left(6+2 \rho\left(L_{0}\right)\right)\right](1+O(\epsilon)) .
\end{gathered}
$$

Here we have used that by symmetry

$$
S_{\epsilon}^{\prime}(0)=0 \text { and } S_{\epsilon}^{\prime}\left(\epsilon_{1} y_{1}\right)=\epsilon_{1} y_{1} S_{\epsilon}^{\prime \prime}(0)+O\left(\epsilon_{1}^{2} y_{1}^{2}\right) .
$$

Similarly, we compute, using the Green's function $G_{D, \tau \lambda_{\epsilon}}$ (see (9.7)), that

$$
\psi_{1, \epsilon}\left(\epsilon_{1} y_{1}\right)-\psi_{1, \epsilon}(0)
$$




$$
\begin{gathered}
=a_{1} \int_{\Omega_{\epsilon_{1}}}\left[G_{D, \tau \lambda_{\epsilon}}\left(\epsilon_{1} y_{1}, \epsilon_{1} z_{1}\right)-G_{D, \tau \lambda_{\epsilon}}\left(0, \epsilon_{1} z_{1}\right)\right] 2 S_{\epsilon}\left(\epsilon_{1} z_{1}\right) u_{1, \epsilon}\left(z_{1}\right) \frac{\epsilon_{2}}{\epsilon_{1}} \tilde{u}_{1, \epsilon}^{\prime}\left(z_{1}\right) d z_{1}\left(1+O\left(\epsilon_{1}\left|y_{1}\right|\right)\right) \\
=a_{1}\left(1+O\left(\epsilon \log \frac{1}{\epsilon}\right)\right)\left[\epsilon_{2} \int_{-1 / \epsilon_{1}}^{1 / \epsilon_{1}} \frac{1}{D}\left(\left|y_{1}-z_{1}\right|-\left|z_{1}\right|\right) S_{\epsilon} u_{1, \epsilon} \frac{1}{\epsilon_{1}} \tilde{u}_{1, \epsilon}^{\prime} d z_{1}\right. \\
+\epsilon_{1} \epsilon_{2} \frac{2}{S_{\epsilon}(0)} \underbrace{\left.\nabla_{x} \nabla_{z} H_{D}(x, z)\right|_{x=y=0}}_{=0} y_{1} \int_{-L_{0}-r_{\epsilon}}^{\infty} z_{1} 2 w w^{\prime} d z_{1}]\left(1+O\left(\left(\tau+\tau_{1}\right)\left|\lambda_{\epsilon}\right|\right)\right) .
\end{gathered}
$$

Note that from (8.6), we derive, using (9.7), that

$$
\psi_{1, \epsilon}(0)=O\left(\epsilon+\left(\tau+\tau_{1}\right)\left|\lambda_{\epsilon}\right|\right) .
$$

Adding (8.9) and (8.11), we get

$$
\begin{gathered}
\frac{d}{d x}\left[S_{\epsilon}\left(\epsilon_{1} y_{1}\right)-S_{\epsilon}(0)\right]-\left[\psi_{\epsilon}\left(\epsilon_{1} y_{1}\right)-\psi_{\epsilon}(0)\right] \\
=-\frac{\epsilon_{1}}{D} \frac{a_{1}}{S_{\epsilon}(0)} y_{1}\left(6+2 \rho\left(L_{0}\right)\right)\left(1+O\left(\epsilon \log \frac{1}{\epsilon}+\left(\tau+\tau_{1}\right)\left|\lambda_{\epsilon}\right|\right)\right) .
\end{gathered}
$$

Suppose that $\left(\phi_{1, \epsilon}, \phi_{2, \epsilon}\right)$ satisfies $\left\|\left|\left(\phi_{1, \epsilon}, \phi_{2, \epsilon}\right) \|\right|_{H^{2}}=1\right.$ for the norms $\| \mid \cdot \|$ introduced in (7.5), (7.6). Then $\left|a^{\epsilon}\right| \leq C$.

Substituting the decompositions of $\psi_{\epsilon}, \phi_{1, \epsilon}$ and $\phi_{2, \epsilon}$ into (5.3) and subtracting (8.3), we have

$$
\begin{gathered}
\tau_{1} \lambda_{\epsilon}\left(a^{\epsilon} \frac{\epsilon_{2}}{\epsilon_{1}} \tilde{u}_{1, \epsilon}^{\prime}\right) \\
=a^{\epsilon} \tilde{u}_{1, \epsilon}^{2}\left(\psi_{1, \epsilon}-\epsilon_{2} S_{\epsilon}^{\prime}\right) \\
+\left(\bar{\phi}_{1, \epsilon}\right)^{\prime \prime}-\bar{\phi}_{1, \epsilon}+2 u_{1, \epsilon} S_{\epsilon} \bar{\phi}_{1, \epsilon}+u_{1, \epsilon}^{2} \bar{\psi}_{\epsilon}-\tau_{1} \lambda_{\epsilon} \bar{\phi}_{1, \epsilon} \\
-a_{2} \frac{\epsilon_{1}}{\epsilon_{2}} \bar{\phi}_{1, \epsilon} u_{2, \epsilon}^{2}-2 a_{2} \frac{\epsilon_{1}}{\epsilon_{2}} u_{1, \epsilon} u_{2, \epsilon} \bar{\phi}_{2, \epsilon}+\text { e.s.t. } \\
=: I_{1}+I_{2}+I_{3} .
\end{gathered}
$$

Let us first compute, using (8.10), (8.12) and (8.13),

$$
\begin{gathered}
I_{1}=a^{\epsilon} \tilde{u}_{1, \epsilon}^{2}\left(\psi_{1, \epsilon}-\epsilon_{2} S_{\epsilon}^{\prime}\right) \\
=\epsilon_{1} \epsilon_{2} a^{\epsilon} \frac{a_{1}}{D S_{\epsilon}(0)} y_{1} u_{1, \epsilon}^{2}\left(6+2 \rho\left(L_{0}\right)\right)\left(1+O\left(\epsilon \log \frac{1}{\epsilon}+\left(\tau+\tau_{1}\right)\left|\lambda_{\epsilon}\right|\right)\right) \\
=c^{\epsilon} \epsilon_{2}^{2} y_{2} u_{1, \epsilon}^{2}\left(1+O\left(\epsilon \log \frac{1}{\epsilon}+\left(\tau+\tau_{1}\right)\left|\lambda_{\epsilon}\right|\right)\right),
\end{gathered}
$$

where

$$
c^{\epsilon}=a^{\epsilon} \frac{a_{1}}{D S_{\epsilon}(0)}\left(6+2 \rho\left(L_{0}\right)\right) .
$$

Since we will see that $\left|\lambda_{\epsilon}\right|=O\left(\epsilon_{2}^{2}\right)$, the second part $\left(\tau+\tau_{1}\right)\left|\lambda_{\epsilon}\right|$ in the error of (8.15) is dominated by its first part $\epsilon \log \frac{1}{\epsilon}$. The same remark applies for the rest of this proof. For brevity, the second part is omitted from now on. 
Next we estimate $I_{2}$. To this end, we decompose $\bar{\phi}_{1, \epsilon}$ into three parts:

$$
\bar{\phi}_{1, \epsilon}=-c^{\epsilon} \epsilon_{1} \epsilon_{2} y_{1} \tilde{u}_{1, \epsilon}+a^{\epsilon}\left(\bar{\phi}_{1 a, \epsilon}-\frac{\epsilon_{2}}{\epsilon_{1}} \tilde{u}_{1, \epsilon}^{\prime}\right)+\bar{\phi}_{1 b, \epsilon} .
$$

We will show that $-c^{\epsilon} \epsilon_{1} \epsilon_{2} y_{1} \tilde{u}_{1, \epsilon}$ is the leading part which gives the main contribution to the small eigenvalues. Further, $\bar{\phi}_{1 a, \epsilon}$ and $\bar{\phi}_{1 b, \epsilon}$ will be introduced and estimated below.

The leading part $-c^{\epsilon} \epsilon_{1} \epsilon_{2} y_{1} \tilde{u}_{1, \epsilon}$ of $\bar{\phi}_{1, \epsilon}$ satisfies

$$
\begin{gathered}
\left(-c^{\epsilon} \epsilon_{1} \epsilon_{2} y_{1} \tilde{u}_{1, \epsilon}\right)^{\prime \prime}-\left(-c^{\epsilon} \epsilon_{1} \epsilon_{2} y_{1} \tilde{u}_{1, \epsilon}\right)+2 \tilde{u}_{1, \epsilon} S_{\epsilon}\left(-c^{\epsilon} \epsilon_{1} \epsilon_{2} y_{1} \tilde{u}_{1, \epsilon}\right) \\
+c^{\epsilon} \epsilon_{1}^{2} \frac{\epsilon_{2}}{\epsilon_{1}} y_{1} \tilde{u}_{1, \epsilon}^{2}+2 c^{\epsilon} \epsilon_{1} \epsilon_{2} \tilde{u}_{1, \epsilon}^{\prime}=O\left(\epsilon_{1}^{2} \epsilon_{2} y_{1}^{2}\right) \quad \text { in } L^{2}\left(\Omega_{\epsilon_{1}}\right) .
\end{gathered}
$$

Thus it introduces the extra term $2 c^{\epsilon} \epsilon_{1} \epsilon_{2} \tilde{u}_{1, \epsilon}^{\prime}$ into $I_{2}$.

We have to cancel this extra term by adding a correction to $\frac{\epsilon_{2}}{\epsilon_{1}} \tilde{u}_{1, \epsilon}^{\prime}$. This is done as follows: Let $\bar{\phi}_{1 a, \epsilon}$ for $\left|y_{2}\right| \leq r_{\epsilon} \frac{\epsilon_{1}}{\epsilon_{2}}$ be defined by

$$
\left(\bar{\phi}_{1 a, \epsilon}\right)_{y_{2}, y_{2}}-\frac{\epsilon_{2}^{2}}{\epsilon_{1}^{2}}\left(1+2 c^{\epsilon} \epsilon_{1}^{2}\right) \bar{\phi}_{1 a, \epsilon}+\frac{\epsilon_{2}^{2}}{\epsilon_{1}^{2}} 2 u_{1, \epsilon} S_{\epsilon} \bar{\phi}_{1 a, \epsilon}-a_{2} \frac{\epsilon_{2}}{\epsilon_{1}}\left[2 u_{1, \epsilon} u_{2, \epsilon} \tilde{u}_{2, \epsilon}^{\prime}+\bar{\phi}_{1 a, \epsilon} u_{2, \epsilon}^{2}\right]=o\left(\epsilon_{2}^{2}\right) .
$$

Note that $\frac{\epsilon_{2}}{\epsilon_{1}} \tilde{u}_{1, \epsilon}^{\prime}$ solves the equation (8.17) except for the term $2 c^{\epsilon} \epsilon_{2}^{2} \bar{\phi}_{1 a, \epsilon}$ (and higher order terms dominated by it). Comparing $\frac{\epsilon_{2}}{\epsilon_{1}} \tilde{u}_{1, \epsilon}^{\prime}$ and $\bar{\phi}_{1 a, \epsilon}$, we get

$$
\left(\bar{\phi}_{1 a, \epsilon}-\tilde{u}_{1, \epsilon, y_{2}}\right)_{y_{2}, y_{2}}-2 c^{\epsilon} \epsilon_{2}^{2} \tilde{u}_{1, \epsilon, y_{2}}=o\left(\epsilon_{2}^{2}\right), \quad\left|y_{2}\right| \leq r_{\epsilon} \frac{\epsilon_{1}}{\epsilon_{2}} .
$$

Integrating the first equation of (8.3) w.r.t. $y_{2}$, we derive

$$
\left|\tilde{u}_{1, \epsilon, y_{2}}\right|=O\left(\frac{\epsilon_{2}}{\epsilon_{1}}\right), \quad\left|y_{2}\right| \leq r_{\epsilon} \frac{\epsilon_{1}}{\epsilon_{2}} .
$$

Inserting into (8.18) and integrating, we get

$$
\left|\bar{\phi}_{1 a, \epsilon}-\tilde{u}_{1, \epsilon, y_{2}}\right| \leq\left|c^{\epsilon}\right| O\left(\frac{\epsilon_{2}^{3}}{\epsilon_{1}} y_{2}^{2}\right), \quad\left|y_{2}\right| \leq r_{\epsilon} \frac{\epsilon_{1}}{\epsilon_{2}} .
$$

By Proposition 7.1 we derive the error estimate

$$
\left\|\left|\left(\bar{\phi}_{1 b, \epsilon}, \phi_{2, \epsilon}^{\perp}\right) \|\right|_{H^{2}}=o\left(\epsilon^{2}\right) .\right.
$$

Using the estimate

$$
\left|\bar{\psi}_{\epsilon}(0)\right|=O\left(\epsilon_{1}^{2} \epsilon_{2}\right)
$$

which follows from (8.7) and (9.7) since $\bar{\phi}_{1, \epsilon}$ is an odd function, we get

$$
I_{2}=o\left(\epsilon_{2}^{2}\right) \quad \text { in } L^{2}\left(\Omega_{\epsilon_{1}}\right) .
$$

Finally, from (8.19) we get

$$
I_{3}=o\left(\epsilon_{2}^{2}\right) \quad \text { in } L^{2}\left(\Omega_{\epsilon_{2}}\right) .
$$

Therefore we derive

$$
\begin{gathered}
\left(\bar{\phi}_{1 a, \epsilon}^{\prime}(0)-\tilde{u}_{1, \epsilon, y_{2}}(0)\right) \frac{\epsilon_{2}}{\epsilon_{1}} y_{2} w^{2}=o\left(\epsilon_{2}^{2}\right) c^{\epsilon} u_{1, \epsilon}(0) y_{2} w^{2}, \\
\bar{\phi}_{1 b, \epsilon}^{\prime}(0) \frac{\epsilon_{2}}{\epsilon_{1}} y_{2} w^{2}=o\left(\epsilon_{2}^{2}\right) c^{\epsilon} u_{1, \epsilon}(0) y_{2} w^{2}
\end{gathered}
$$


and so

$$
\bar{\phi}_{1, \epsilon}^{\prime}(0) \frac{\epsilon_{2}}{\epsilon_{1}} y_{2} w^{2}=\epsilon_{2}^{2} c^{\epsilon} u_{1, \epsilon}(0) y_{2} w^{2}(1+o(1)) .
$$

Multiplying the second component of the eigenvalue problem (5.3) by $w^{\prime}$ and integrating, we get

$$
\begin{gathered}
\text { l.h.s. }=\epsilon_{2}^{2} c^{\epsilon} u_{1, \epsilon}(0)\left(1+O\left(\epsilon \log \frac{1}{\epsilon}\right)\right) \int_{\mathbb{R}} y_{2} w^{2} w^{\prime} d y_{2} \\
=-\epsilon_{2}^{2} c^{\epsilon} u_{1, \epsilon}(0)\left(1+O\left(\epsilon \log \frac{1}{\epsilon}\right)\right) \int_{\mathbb{R}} \frac{w^{3}}{3} d y_{2} \\
=-2.4 \epsilon_{2}^{2} c^{\epsilon} u_{1, \epsilon}(0)\left(1+O\left(\epsilon \log \frac{1}{\epsilon}\right)\right) \\
=-4.8 \epsilon_{2}^{2} a^{\epsilon} \frac{a_{1}}{D S_{\epsilon}(0)} u_{1, \epsilon}(0)\left(3+\rho\left(L_{0}\right)\right)\left(1+O\left(\epsilon \log \frac{1}{\epsilon}\right)\right) \\
=-4.8 \epsilon_{2}^{2} a^{\epsilon} \frac{a_{1}}{D S_{\epsilon}^{2}(0)} w\left(-L_{0}\right)\left(3+\rho\left(L_{0}\right)\right)(1+o(1))
\end{gathered}
$$

and

$$
\begin{gathered}
\text { r.h.s. }=\lambda_{\epsilon} a^{\epsilon} \int_{\mathbb{R}}\left(w^{\prime}\right)^{2} d y_{2}\left(1+O\left(\epsilon \log \frac{1}{\epsilon}\right)\right) \\
=1.2 a^{\epsilon} \lambda_{\epsilon}(1+o(1)) .
\end{gathered}
$$

Therefore

$$
\begin{aligned}
\lambda_{\epsilon}= & -4 \epsilon_{2}^{2} \frac{a_{1}}{D S_{\epsilon}^{2}(0)} w\left(-L_{0}\right)\left(3+\rho\left(L_{0}\right)\right)+o\left(\epsilon_{2}^{2}\right) \\
& =-12 \epsilon_{2}^{2} \frac{a_{1} a_{2}}{D} \frac{(z+1)(2-z)}{z(1-z)}+o\left(\epsilon_{2}^{2}\right) .
\end{aligned}
$$

We summarize our result on the small eigenvalues in the following theorem.

Theorem 8.1. The eigenvalues of (5.1) with $\lambda_{\epsilon} \rightarrow 0$ satisfy

$$
\lambda_{\epsilon}=-12 \epsilon_{2}^{2} \frac{a_{1} a_{2}}{D} \frac{(z+1)(2-z)}{z(1-z)}+o\left(\epsilon_{2}^{2}\right),
$$

where $z$ has been introduced in (5.16). In particular these eigenvalues are stable.

This completes the proof of Theorem 3.2. 


\section{Appendix C: Two Green's Functions}

Let $G_{D}(x, z)$ be the Green's function of the Laplace operator with Neumann boundary conditions:

$$
\begin{cases}D G_{D}^{\prime \prime}(x, z)+\frac{1}{2}-\delta_{z}=0 & \text { in }(-1,1), \\ \int_{-1}^{1} G_{D}(x, z) d x=0, G_{D}^{\prime}(-1, z)=G_{D}^{\prime}(1, z)=0 . & \end{cases}
$$

We can decompose $G_{D}(x, z)$ as follows

$$
G_{D}(x, z)=\frac{1}{2 D}|x-z|+H_{D}(x, z)
$$

where $H_{D}$ is the regular part of $G_{D}$.

Written explicitly, we have

$$
G_{D}(x, z)= \begin{cases}\frac{1}{D}\left[\frac{1}{3}-\frac{(x+1)^{2}}{4}-\frac{(1-z)^{2}}{4}\right], & -1<x \leq z \\ \frac{1}{D}\left[\frac{1}{3}-\frac{(z+1)^{2}}{4}-\frac{(1-x)^{2}}{4}\right], & z \leq x<1\end{cases}
$$

By simple computations,

$$
H_{D}(x, z)=\frac{1}{2 D}\left[-\frac{1}{3}-\frac{x^{2}}{2}-\frac{z^{2}}{2}\right] .
$$

For $x \neq z$ we calculate

$$
\nabla_{x} \nabla_{z} G_{D}(x, z)=0, \quad \nabla_{x} G_{D}(x, z)= \begin{cases}-\frac{x+1}{2 D} & \text { if } x \leq z \\ -\frac{x-1}{2 D} & \text { if } z \leq x .\end{cases}
$$

We further have

$$
\left.\nabla_{x} G_{D}(x, z)\right|_{x=z}=\left.\nabla_{x} H_{D}(x, z)\right|_{x=z}=-\frac{z}{2 D}
$$

(see [12]).

Similarly, let $G_{D, \tau \lambda_{\epsilon}}(x, z)$ be Green's function of

$$
\left\{\begin{array}{l}
D G_{D, \tau \lambda_{\epsilon}}^{\prime \prime}(x, z)-\tau \lambda_{\epsilon} G_{D, \tau \lambda_{\epsilon}}(x, z)-\delta_{z}=0 \quad \text { in }(-1,1) \\
G_{D, \tau \lambda_{\epsilon}}^{\prime}(-1, z)=G_{D, \tau \lambda_{\epsilon}}^{\prime}(1, z)=0
\end{array}\right.
$$

We can decompose $G_{D, \tau \lambda_{\epsilon}}(x, z)$ as follows

$$
G_{D, \tau \lambda_{\epsilon}}(x, z)=\frac{1}{2 D}|x-z|+H_{D, \tau \lambda_{\epsilon}}(x, z)
$$

where $H_{D, \tau \lambda_{\epsilon}}$ is the regular part of $G_{D, \tau \lambda_{\epsilon}}$.

An elementary computation shows that

$$
\left|H_{D}(x, z)-H_{D, \tau \lambda}(x, z)\right| \leq C\left|\tau \lambda_{\epsilon}\right|
$$

uniformly for all $(x, z) \in \Omega \times \Omega$.

Acknowledgments: This research is supported by an Earmarked Research Grant from RGC of Hong Kong. MW thanks the Department of Mathematics at The Chinese University of Hong Kong for their kind hospitality. 


\section{REFERENCES}

[1] K. Amonlirdviman, N. A. Khare, D. R. P. Tree, W. Chen, J. D. Axelrod and C. J. Tomlin, Mathematical modeling of planar cell polarity to understand domineering nonautonomy, Science 307 (2005), 423-426.

[2] D. L. Benson, P. K. Maini, and J. A. Sherratt, Unravelling the Turing bifurcation using spatially varying diffusion coefficients, J. Math. Biol. 37 (1998), 381-417.

[3] L. Bettencourt and G. West, A unified theory of urban living, Nature 467 (2010), 912-913.

[4] E.N. Dancer, On stability and Hopf bifurcations for chemotaxis systems, Methods Appl. Anal. 8 (2001), 245-256.

[5] A. Doelman, A. Gardner and T.J. Kaper, Stability analysis of singular patterns in the 1-D Gray-Scott model: A matched asymptotic approach, Phys. D 122 (1998), 1-36.

[6] A. Doelman, T. Kaper and P. A. Zegeling, Pattern formation in the one-dimensional Gray-Scott model, Nonlinearity 10 (1997), 523-563.

[7] A. Doelman, R. Gardner and T. J. Kaper, Large stable pulse solutions in reaction-diffusion equations, Indiana Univ. Math. J. 50, 443-507 (2001).

[8] M. Eigen and P. Schuster, The hypercycle: A principle of natural selforganisation (Springer, Berlin, 1979).

[9] A. Gierer and H. Meinhardt, A theory of biological pattern formation, Kybernetik (Berlin) 12 (1972), 30-39.

[10] P. Gray and S.K. Scott, Autocatalytic reactions in the isothermal, continuous stirred tank reactor: isolas and other forms of multistability, Chem. Eng. Sci. 38 (1983), 29-43.

[11] P. Gray and S.K. Scott, Autocatalytic reactions in the isothermal, continuous stirred tank reactor: oscillations and instabilities in the system $A+2 B \rightarrow 3 B, B \rightarrow C$, Chem. Eng. Sci. 39 (1984), 10871097.

[12] D. Iron, J. Wei and M. Winter, Stability analysis of Turing patterns generated by the Schnakenberg model, J. Math. Biol. 49 (2004), 358-390.

[13] A. Klaus and W. Birchmeier, Wnt signalling and its impact on development and cancer, Nature Reviews Cancer 8 (2008), 387-398.

[14] P.K. Maini, J. Wei and M. Winter, Stability of spikes in the shadow Gierer-Meinhardt system with Robin boundary conditions, Chaos 17 (2007), 037106.

[15] H. Meinhardt and A. Gierer, Generation and regeneration of sequences of structures during morphogenesis, J. Theor. Biol., 85 (1980), 429-450.

[16] J.D. Murray, Mathematical Biology II: Spatial Models and Biomedical Applications, Interdisciplinary Applied Mathematics Vol. 18, Springer, 2003.

[17] W.-M. Ni and I. Takagi, On the shape of least energy solution to a semilinear Neumann problem, Comm. Pure Appl. Math. 41 (1991), 819-851.

[18] W.-M. Ni and I. Takagi, Locating the peaks of least energy solutions to a semilinear Neumann problem, Duke Math. J. 70 (1993), 247-281.

[19] W.-M. Ni and I. Takagi, Point-condensation generated by a reaction-diffusion system in axially symmetric domains, Japan J. Industrial Appl. Math. 12 (1995), 327-365.

[20] Y. Nishiura and D. Ueyama, A skeleton structure of self-replicating dynamics, Physcia D 130 (1999), 73-104.

[21] Y. Nishiura, T. Teramoto and D. Ueyama, Scattering and separators in dissipative systems, Phys. Rev. E 67 (2003), 056210.

[22] J. Schnakenberg, Simple chemical reaction systems with limit cycle behaviour, J. Theoret. Biol. 81 (1979), 389-400.

[23] I. Takagi, Point-condensation for a reaction-diffusion system, J. Differential Equations 61 (1986), 208249.

[24] A. M. Turing, The chemical basis of morphogenesis, Phil. Trans. Roy. Soc. Lond. B 237 (1952), 37-72. 
[25] A. I. Volpert, Vitaly A. Volpert and Vladimir A. Volpert, Traveling Wave Solutions of Parabolic Systems, Translations of Mathematical Monographs Vol. 140, American Mathematical Society, 1994.

[26] M.J. Ward and J. Wei, Asymmetric spike patterns for the one-dimensional Gierer-Meinhardt model: equilibria and stability, Europ. J. Appl. Math. 13 (2002), 283-320.

[27] J. Wei, On single interior spike solutions of Gierer-Meinhardt system: uniqueness, spectrum estimates and stability analysis, Euro. J. Appl. Math. 10 (1999), 353-378.

[28] J. Wei, Existence, stability and metastability of point condensation patterns generated by Gray-Scott system, Nonlinearity 12 (1999), 593-616.

[29] J. Wei, On two dimensional Gray-Scott model: existence of single pulse solutions and their stability, Phys. D, 148 (2001), 20-48.

[30] J. Wei and M. Winter, On a two dimensional reaction-diffusion system with hypercyclical structure, Nonlinearity 13 (2000), 2005-2032.

[31] J. Wei and M. Winter, Spikes for the two-dimensional Gierer-Meinhardt system: The weak coupling case, J. Nonlinear Science 6 (2001), 415-458.

[32] J. Wei and M. Winter, Critical threshold and stability of cluster solutions for large reaction-diffusion systems in $\mathbb{R}^{1}$, SIAM J. Math Anal.

[33] J. Wei and M. Winter, Existence, classification and stability analysis of multiple-peaked solutions for the Gierer-Meinhardt system in $\mathbb{R}^{1}$, Methods Appl. Anal. 14 (2007), 119-164.

[34] J. Wei and M. Winter, Stationary multiple spots for reaction-diffusion systems, J. Math. Biol. 57 (2008), 53-89.

[35] J. Wei and M. Winter, Mutually exclusive spiky pattern and segmentation modeled by the fivecomponent Meinhardt-Gierer system, SIAM J. Appl. Math. 69 (2008), 419-452.

[36] J. Wei and M. Winter, Spikes for the Gierer-Meinhardt system with discontinuous diffusion coefficients, J. Nonlinear Sci. 19 (2009), 301-339. 